Federal Reserve Bank of Minneapolis

Research Department Staff Report 258

February 1999

\title{
Sequential Equilibria in a Ramsey Tax Model*
}

\author{
Christopher Phelan
}

Federal Reserve Bank of Minneapolis

and Kellogg Graduate School of Management, Northwestern University

Ennio Stacchetti

University of Michigan

\begin{abstract}
This paper presents a full characterization of the equilibrium value set of a Ramsey tax model. More generally, it develops a dynamic programming method for a class of policy games between the government and a continuum of consumers. By selectively incorporating Euler conditions into a strategic dynamic programming framework, we wed two technologies that are usually considered competing alternatives, resulting in a dramatic simplification of the problem.

*This research was supported in part by the National Science Foundation. First draft: November 1996. The views expressed herein are those of the authors and not necessarily those of the Federal Reserve Bank of Minneapolis or the Federal Reserve System.
\end{abstract}




\section{Introduction}

This paper presents a full characterization of the equilibrium value set of a Ramsey tax model. More generally, it develops a dynamic programming method for a class of policy games between the government and a continuum of consumers.

Starting with the seminal paper of Kydland and Prescott (1977), there is by now a large body of literature dealing with reputation and credibility of government policies (see, for example, Chari and Kehoe (1990, 1993a, 1993b), Persson, Persson and Svensson (1987), Rogoff (1989), and Stokey (1989, 1991)). A main concern of this work is to determine, for example, the extent to which the government can credibly borrow money and/or commit to maximal levels of capital taxes. Most of these papers have partially adapted dynamic programming techniques developed for repeated games, to deal with a situation involving strategically anonymous players, and (sometimes) stock variables. Because of the heightened complexity associated with the state variables and the continuum of agents, most papers have dealt with drastically simplified models and have restricted attention to rather crude punishment strategies.

One of the main thrusts of the paper by Kydland and Prescott (1977) was that the inability of governments to commit to policies made the application of optimal control techniques to the government's optimization problem inappropriate. One must instead examine policy in the context of a dynamic game. Thus, the approach of Abreu, Pearce and Stacchetti $(1986,1990)$ is a natural starting point for a full analysis of most policy games. Atkeson (1991) and others have already demonstrated that strategic dynamic programming can in principle incorporate the presence of state variables (such as stocks of capital or debt) and can yield useful characterizations in special cases. More generally, tractability becomes a 
major concern.

A direct extension of Abreu, Pearce and Stacchetti's results to the macro policy game setting would require that each consumer (in the continuum of agents in the economy) be assigned a continuation value after each history, in such a way that these values provide incentives for conforming to equilibrium behavior, even once a consumer has the "wrong" level of personal assets because she did not conform in the past. In general this produces a problem that is prohibitively complex, either for the derivations of interesting properties or for numerical computations.

We propose instead a much simpler accounting system, wherein we only keep track of the "marginal value of capital" of an "honest" consumer and use the "convexity" of the consumers' dynamic consumption problem to neglect completely the incentives of dishonest consumers. The crucial observation is that since each agent (other than the government) is anonymous and does not affect the path of prices, for any given price path, the household's problem, unlike that of the government, can be treated by standard optimal control theory. By selectively incorporating Euler conditions into the strategic dynamic programming framework, we wed two technologies that are usually considered competing alternatives. The result is a dramatic simplification of the problem.

The specific model we consider is a one-sector growth model with capital and labor, as well as a public good which must be financed by distortionary taxes on capital and labor. The government is unable to commit to future tax rates. Any tax policy by the government produces a dynamic economy, and associated with each tax policy there is a Walrasian equilibrium. In a similar model, Chari and Kehoe (1990) propose a method for checking 
whether a government's tax policy is the outcome of a symmetric sequential equilibrium: ${ }^{1}$ in every period the government should not be able to improve the total future welfare of a representative consumer by a change in the current period's tax rates, if this is then followed by the worst sequential equilibrium of the ensuing subgame. There are two difficulties with this approach. First, it assumes that one can easily construct a worst equilibrium. For many interesting models (in particular, for ours), a worst equilibrium is not easy to obtain. Second, this method provides no guidance on how to generate candidate tax policies. The method is very effective if all one wants to do is to check whether the first-best solution can be sustained in equilibrium.

In contrast, our method deals directly with the whole set of equilibrium values. We are then able to characterize second-best tax policies when first-best policies are unattainable because the discount factor is not sufficiently high. Another advantage of our approach is that it allows us to examine the credibility of the punishments used in equilibrium. In particular, we investigate the steady state of second-best equilibria. A celebrated result of Chamley (1986) and Judd (1985) states that with full commitment, the optimal capital tax rate converges to 0 in steady state. In our anonymous game (without commitment), when the first-best tax policy is not sustainable in a sequential equilibrium, if the second-best policy leads to a steady state in the limit, the capital tax rate remains bounded away from 0 . The limit steady state maintains a constant capital stock $k^{s}$. It turns out that the limit steady state policy is a worst sustainable policy in the economy where the consumers' initial capital stock is $k^{s}$. This may seem paradoxical at first: Why should the continuation of a best

\footnotetext{
${ }^{1}$ Chari and Kehoe (1990) call such an equilibrium a sustainable equilibrium. We prefer to keep the terminology from game theory, as the class of anonymous "games" investigated here can be viewed as an extension of the standard class of dynamic games.
} 
sustainable policy be a worst sustainable policy? We show that, starting with the capital stock $k^{s}$, the limit policy is in a sense both a worst and a best sustainable policy. It is a best equilibrium policy subject to the constraint that the resulting marginal value of capital in the first period is above a certain critical level, but it is a worst policy if the government is free from such a constraint. The critical level of the marginal value of capital is such that, starting with $k^{s}$, if the agents expect the same marginal value of capital in the second period, their savings match the capital depreciation, and the level of capital is back to $k^{s}$ at the end of the period.

Finally, we extend the algorithm proposed by Abreu, Pearce and Stacchetti (1990) for infinitely repeated games with a finite number of players to our current dynamic anonymous game. We have implemented the algorithm on the computer and explicitly calculated the equilibrium value correspondence for various parameter specifications. Conklin and Judd (1996) propose a similar algorithm for dynamic games with a finite number of players. While they use an "internal" approximation to the value correspondence, our computer implementation uses an "external" approximation.

Two other papers deserve particular mention. First, independent work of Chang (1998) considers a monetary model without a physical state and derives methods similar to those developed here. Second, the work of Benhabib and Rustichini (1997) characterizes the best equilibrium of a similar game without commitment using non-recursive methods.

We introduce the model and the assumptions in Section 2. Section 3 characterizes the Walrasian equilibria of the dynamic economy where the government tax policy is fixed (but not necessarily stationary). In Section 4 we modify the game and introduce a public randomization device. This is a standard modification to ensure that equilibrium value sets 
are convex and thus avoid some technical difficulties. In Section 5 we define a sequential equilibrium for our anonymous game and extend the dynamic programming tools developed by Abreu, Pearce and Stacchetti (1990) for repeated games. We thus are able to characterize the equilibrium value correspondence. In Section 6 we study the steady state of second-best equilibria. Section 7 presents an example, and Section 8 concludes.

\section{The Game}

We consider a dynamic game with a benevolent government and a continuum of identical households, represented by the interval $[0,1] .^{2}$ There is a single consumption good that is produced with a constant returns to scale technology. If capital and labor per capita are $K$ and $L$, then output per capita is $f(K, L)$. At the beginning of every period $t$, each household is endowed with 1 unit of labor-leisure, and the government chooses the capital tax rate $\tau_{k, t}$ and labor tax rate $\tau_{\ell, t}$ in the interval $[\underline{\tau}, \bar{\tau}]$. Then the households simultaneously choose their labor input. Given the aggregate capital (invested in the previous period) and labor input, the market determines competitive prices for the consumption good (numeraire), capital, and labor. Finally, each household independently chooses consumption and the amount of the good it stores (capital) for production in the next period. Capital fully depreciates each period, ${ }^{3}$ and the totality of tax revenue is used by the government to finance a public good period by period.

If a household consumes the stream $\left\{\left(\ell_{t}, c_{t}, G_{t}\right)\right\}_{t=0}^{\infty}$ of labor, consumption good, and

\footnotetext{
${ }^{2}$ The interval $[0,1]$ is endowed with the standard Lebesgue measure.

${ }^{3}$ We make this assumption to keep the analysis simple; our results can be extended to the case where the depreciation rate is less than 1 and investments are irreversible.
} 
public good per capita, then its total discounted utility is

$$
\sum_{t=0}^{\infty} \beta^{t}\left[u\left(\ell_{t}, c_{t}\right)+g\left(G_{t}\right)\right],
$$

where $\beta \in(0,1)$ is the discount factor. All households have the same preferences. Households are strategically anonymous; that is, their individual actions cannot be observed, and the government can only react to its own past actions and to past distributions of consumption, capital and labor, all of which are publicly observed. The government's objective is to maximize the total discounted utility of a representative household.

Let $\Gamma\left(k_{0}\right)$ denote the dynamic game between the government and the households when all the households, except perhaps for a subset of measure 0, are endowed with initial capital of $k_{0}$. Since we restrict attention to symmetric strategy equilibria (where all households make the same choices along the equilibrium path), we will not need to explicitly consider situations where at the beginning of a period there is a nontrivial distribution of capital.

Assumptions: The following assumptions will be in force throughout the paper:

(A1) $0 \leq \underline{\tau}<\bar{\tau}<1$. $^{4}$

(A2) $g: \mathbf{R}_{+} \rightarrow \mathbf{R}$ and $u:[0,1) \times \mathbf{R}_{+} \rightarrow \mathbf{R}$ are continuously differentiable, $g$ is concave and increasing, and $u(\ell, c)$ is concave (jointly in $\ell$ and $c$ ), increasing in $c$, and decreasing in $\ell$. Moreover, $g(0)=0$, and there exists $U_{c} \in \mathbf{R}$ such that for all $\ell \in[0,1)$ and $c \in \mathbf{R}_{+}$,

$$
u_{c}(\ell, 0) \leq U_{c}, \quad \inf _{\ell^{\prime} \in[0,1)} u_{c}\left(\ell^{\prime}, c\right)>0, \quad \text { and } \quad u_{\ell}(0,0)=0
$$

\footnotetext{
${ }^{4}$ It is possible to choose $\underline{\tau}<0$. But our model does not allow for the possibility of the government borrowing or lending, and thus the government's revenue must be nonnegative in every period. Hence, if $\underline{\tau}<0$, the government could, for example, subsidize labor with revenues from the capital tax. However, this introduces an additional difficulty, which we have preferred to avoid. Since tax rates are announced before the households make their decisions, it is possible that when the government is prepared to subsidize labor (or capital), it doesn't collect enough tax revenue to keep up its promise. Thus, to allow for $\underline{\tau}<0$, we would have to choose some form of rationing for those contingencies.
} 
(A3) $f: \mathbf{R}_{+}^{2} \rightarrow \mathbf{R}_{+}$is strictly concave and homogeneous of degree 1 and is continuously differentiable in the interior of $\mathbf{R}_{+}^{2}$. For all $(k, \ell) \in \operatorname{int}\left(\mathbf{R}_{+}^{2}\right), f(0, \ell)=f(k, 0)=0$, $f_{k}(k, \ell)>0$ and $f_{\ell}(k, \ell)>0$. Moreover, for all $\ell \in[0,1]$,

$$
\lim _{k \rightarrow 0} f_{k}(k, \ell)=\infty \quad \text { and } \quad \lim _{k \rightarrow \infty} f_{k}(k, \ell)=0 .
$$

The assumption that $\bar{\tau}<1$ precludes a particularly bad equilibrium. If $\bar{\tau}=1$, the households may think after a deviation by the government that the government intends to impose capital taxes of $100 \%$ next period. With those beliefs, they would optimally choose to make no capital investments, which in the case of full depreciation would result in a capital stock equal to 0 . Then, by (A3), output would be 0 in every future period, and what taxes are eventually imposed by the government would be irrelevant: the economy would cease to exist. Although this bad outcome satisfies the sequential equilibrium requirements, we believe it is not plausible. Intuitively, one feels this equilibrium lacks renegotiation-proofness. In the absence of any straightforward generalization to dynamic games of the definition of renegotiation-proofness in repeated games suggested by Pearce (1987), we simply eliminate this equilibrium by imposing a gross upper bound on capital tax rates.

In assumption $(\mathrm{A} 2), u_{\ell}(0,0)=0$ is required to ensure that in equilibrium, labor and consumption are positive. The condition $u_{c}(\ell, 0) \leq U_{c}$ guarantees that for the household's optimization problem, any path of (labor, consumption, investment) that is locally optimal also satisfies a transversality condition (see Lemma 4 below). Thus, local optimality is sufficient for global optimality. The function $u$ is assumed to be continuously differentiable only in $[0,1) \times \mathbf{R}_{+}$to allow for the possibility that as $\ell \rightarrow 1$, either $u(\ell, c)$ or $u_{\ell}(\ell, c)$ tends to $-\infty$. However, we require that the marginal utility of consumption for a fixed $c$ is uniformly 
bounded away from 0 for $\ell \in[0,1)$.

Assumption (A3) implies that in a competitive equilibrium, the firm(s) operates at zero profits, and thus it is unnecessary to specify ownership. It also implies that competitive prices in a period are completely determined by the supply of capital and labor. Thus, if in period $t$, the capital and labor per capita are respectively $K_{t}$ and $L_{t}$, then

$$
p_{k, t}=f_{k}\left(K_{t}, L_{t}\right) \quad \text { and } \quad p_{\ell, t}=f_{\ell}\left(K_{t}, L_{t}\right)
$$

The second part of (A3) implies that for any fixed $\ell \in[0,1]$, there exists $\hat{k}$ such that $f(k, \ell)>k$ for all $k<\hat{k}$ and $f(k, \ell)<k$ for all $k>\hat{k}$, and the graph of $f(\cdot, \ell)$ crosses the 45 degree line at $\hat{k}$. The standard Cobb-Douglas production function satisfies (A3).

Definition: Let $\bar{k}$ be the maximal sustainable capital stock in steady state. That is, $\bar{k}$ is the solution of the equation $k=f(k, 1)$ (when labor supply is maximal). $\bar{k}$ also represents the maximal possible output when the initial capital is no more than $\bar{k}$.

Unfortunately, the first part of (A2) (that marginal utility of consumption at $c=0$ is bounded) rules out some commonly used utility functions. We need this assumption in Lemmas 3 and 4 below. However, these lemmas also hold under different assumptions. For example, we can show that their conclusions are valid for the following pair of utility and production functions:

$$
\begin{aligned}
u(\ell, c) & =v(\ell)+\frac{c^{1-\psi}}{1-\psi} \quad(0<\psi<1), \\
f(k, \ell) & =A\left[\alpha k^{\rho}+(1-\alpha) \ell^{\rho}\right]^{\frac{1}{\rho}} \quad(A>0,0<\alpha<1, \rho \leq 0),
\end{aligned}
$$

where $v:[0,1) \rightarrow \mathbf{R}$ is a decreasing, concave function, with $\lim _{\ell \rightarrow 1} v(\ell)=-\infty$. (Note that when $\rho=0, f(k, \ell)=A k^{\alpha} \ell^{1-\alpha}$.) 
In this paper we study exclusively symmetric strategy profiles, where all households choose the same actions (along the equilibrium path). A symmetric strategy profile for $\Gamma\left(k_{0}\right)$ is a pair of strategies $\sigma=\left(\sigma_{C}, \sigma_{G}\right)$. For each $t$, the strategy $\sigma_{G}$ for the government specifies $\left(\tau_{k, t}, \tau_{\ell, t}\right) \in[\underline{\tau}, \bar{\tau}]^{2}$ as a function $\sigma_{G}(t)$ of the publicly observed history $h^{t-1}:=\left(h_{0}, \ldots, h_{t-1}\right)$, where

$$
h_{s}:=\left(\tau_{k, s}, \tau_{\ell, s}, \ell_{s}, c_{s}, k_{s+1}\right), \quad s=0, \ldots, t-1
$$

(When $t=0, h^{-1}=\emptyset$ is the empty history.) In this notation, $\left(\ell_{s}, c_{s}, k_{s+1}\right)$ specifies the average values of labor, consumption, and saving at date $s$. Similarly, the strategy $\sigma_{C}$ for the households (or consumers), specifies $\ell_{t}$ and $\left(c_{t}, k_{t+1}\right)$ as a function of the expanded histories $\left(h^{t-1}, \tau_{k, t}, \tau_{\ell, t}\right)$ and $\left(h^{t-1}, \tau_{k, t}, \tau_{\ell, t}, p_{k, t}, p_{\ell, t}\right)$ respectively, where $\left(p_{k, t}, p_{\ell, t}, 1\right)$ is the competitive price vector for capital, labor, and the consumption good in period $t$. The choices available to the household are limited by the nonnegativity constraints and the budget constraint:

$$
\begin{aligned}
& 0 \leq \ell_{t} \leq 1, \quad 0 \leq c_{t}, \quad 0 \leq k_{t+1}, \quad \text { and } \\
& c_{t}+k_{t+1}=\left(1-\tau_{k, t}\right) p_{k, t} k_{t}+\left(1-\tau_{\ell, t}\right) p_{\ell, t} \ell_{t} .
\end{aligned}
$$

Let $\Sigma\left(k_{0}\right)=\Sigma_{C}\left(k_{0}\right) \times \Sigma_{G}\left(k_{0}\right)$ denote the set of all symmetric strategy profiles for $\Gamma\left(k_{0}\right)$.

A strategy profile $\sigma \in \Sigma\left(k_{0}\right)$ inductively generates a unique outcome path (or trajectory) $\left\{\left(\tau_{k, t}, \tau_{\ell, t}, \ell_{t}, c_{t}, k_{t+1}\right)\right\}_{t=0}^{\infty}$ for the economy as follows. For each $t \geq 0$, let

$$
\begin{aligned}
\left(\tau_{k, t}, \tau_{\ell, t}\right) & :=\sigma_{G}(t)\left(h^{t-1}\right) \quad \text { and } \quad \ell_{t}:=\sigma_{C 1}(t)\left(h^{t-1}, \tau_{k, t}, \tau_{\ell, t}\right), \\
p_{k, t} & :=f_{k}\left(k_{t}, \ell_{t}\right) \quad \text { and } \quad p_{\ell, t}:=f_{\ell}\left(k_{t}, \ell_{t}\right), \\
\left(c_{t}, k_{t+1}\right) & :=\sigma_{C 2}(t)\left(h^{t-1}, \tau_{k, t}, \tau_{\ell, t}, p_{k, t}, p_{\ell, t}\right), \text { and }
\end{aligned}
$$




$$
h^{t}:=\left(h^{t-1}, \tau_{k, t}, \tau_{\ell, t}, \ell_{t}, c_{t}, k_{t+1}\right) .
$$

The marginal value of capital associated with $\sigma$ is

$$
\Phi_{C}\left(k_{0}, \sigma\right):=\left(1-\tau_{k, 0}\right) f_{k}\left(k_{0}, \ell_{0}\right) u_{c}\left(\ell_{0}, c_{0}\right)
$$

This represents the increase in the household's utility had it started with an additional unit of capital and spent all the additional income on consumption in period 0 . The value of $\sigma$ (that is, the government's normalized total discounted payoff) is ${ }^{5}$

$$
\Phi_{G}\left(k_{0}, \sigma\right):=(1-\beta) \sum_{t=0}^{\infty} \beta^{t}\left[u\left(\ell_{t}, c_{t}\right)+g\left(\tau_{k, t} p_{k, t} k_{t}+\tau_{\ell, t} p_{\ell, t} \ell_{t}\right)\right]
$$

Finally, we let $\Phi\left(k_{0}, \sigma\right):=\left(\Phi_{C}\left(k_{0}, \sigma\right), \Phi_{G}\left(k_{0}, \sigma\right)\right)$.

As formulated above, a symmetric strategy profile is an incomplete specification of the strategies for the government and households. First, it does not specify behavior for a household that has deviated in the past and thus may have a different level of capital than the other households. Second, by specifying the history in terms of past mean levels of consumption, labor, and capital, we are not allowing the government's strategy to depend on deviations by households which affect an observed distribution but not its mean. However, our analysis concerns sequential equilibria only, where multiple deviations are irrelevant for checking the incentives of the players. Since a deviation by a single household is unobservable by the government (and the other households), it does not change the public history, and thus it does not change the future behavior of the government or the other households. Hence, when checking the incentives of a deviant household, we will assume that the outcome path

\footnotetext{
${ }^{5}$ The normalization translates the discounted sum of payoffs into a weighted average, with weights $(1-\beta) \beta^{t}$ that add up to 1 . This makes the value of $\sigma$ comparable with the payoffs received in any period and simplifies our notation below. However, we do not normalize the payoffs of the household because with normalization, the marginal value of capital would have to be multiplied by the factor $(1-\beta)$.
} 
(in particular, taxes and prices) remain the same and that the deviant household optimizes its future intertemporal choices given its own capital stock. It is precisely the inability of a household to unilaterally change the history that allows us to express its incentive constraints with appropriate Euler conditions. Similarly, simultaneous deviations that affect observable distributions but not their means are irrelevant, and, without loss of generality, we can restrict attention to strategies that only react to the type of histories we have specified. Further, we could restrict attention to strategies that only depend on the past history of government's decisions and define $h_{s}$ to be just $\left(\tau_{k, s}, \tau_{\ell, s}\right)$. However, we keep our original notation as a simpler bookkeeping device.

A household makes its decision of labor input immediately after knowing the tax rates. While no household sees itself as affecting the wage rate or the rental rate of capital, the common choice of labor input determines these values, and thus the household budget set regarding the consumption/savings decision as well. However, since the government does not get to move between the households' decisions of labor input and the households' consumption/savings decisions, it will be convenient sometimes to view the strategy $\sigma_{C}$ for the household as if it determined $\left(\ell_{t}, c_{t}, k_{t+1}\right)$ simultaneously as a function of $\left(h^{t-1}, \tau_{k, t}, \tau_{\ell, t}\right)$. In this case we will simply write $\left(\ell_{t}, c_{t}, k_{t+1}\right)=\sigma_{C}(t)\left(h^{t-1}, \tau_{k, t}, \tau_{\ell, t}\right)$.

The following technical lemma will be useful to obtain several bounds. We will assume later that the initial capital stock $k_{0}$ is less than or equal to $\bar{k}$. In this case, the lemma establishes that the sequence of capital stocks $\left\{k_{t}\right\}$ generated by any symmetric strategy profile is uniformly bounded above by $\bar{k}$.

Lemma 1. Let $\sigma$ be a symmetric strategy profile for $\Gamma\left(k_{0}\right)$, with outcome path 
$\left\{\left(\tau_{k, t}, \tau_{\ell, t}, \ell_{t}, c_{t}, k_{t+1}\right)\right\}_{t=0}^{\infty}$. Then, $k_{t} \leq \max \left\{k_{0}, \bar{k}\right\}$ for all $t \geq 1$

Proof. Consider the situation where every household spends all its time working and reinvests all the output it gets (that is, it consumes nothing). Obviously, the sequence of capitals (per capita) $\left\{K_{t}\right\}_{t=0}^{\infty}$ generated by this process bounds the sequence $\left\{k_{t}\right\}_{t=0}^{\infty}$ generated by $\sigma$. That is, $0 \leq k_{t} \leq K_{t}$. By (A3), we also have that for each $t \geq 0$,

$$
K_{t+1}=f\left(K_{t}, 1\right) \leq \max \left\{K_{t}, \bar{k}\right\} \leq \max \left\{K_{0}, \bar{k}\right\}
$$

More specifically, if $K_{0} \leq \bar{k}$, the sequence $\left\{K_{t}\right\}$ increases monotonically to $\bar{k}$, and if $K_{0} \geq \bar{k}$, the sequence decreases monotonically to $\bar{k}$.

For each $t \geq 0$, let $H\left(k_{0}, t\right)$ denote the collection of all $(t+1)$-period histories $h^{t}$ that can be generated by some symmetric strategy profile $\sigma$ of $\Gamma\left(k_{0}\right)$. It is also convenient to define $H\left(k_{0},-1\right):=\{\emptyset\}$ and $H\left(k_{0}\right):=\bigcup_{t=-1}^{\infty} H\left(k_{0}, t\right)$. For any $h^{t} \in H\left(k_{0}, t\right)$, let $\kappa\left(k_{0}, h^{t}\right)$ denote the current capital stock after history $h^{t}$. That is, if $h^{t}=\left(h_{0}, \ldots, h_{t}\right)$ and $h_{t}=$ $\left(\tau_{k, t}, \tau_{\ell, t}, \ell_{t}, c_{t}, k_{t+1}\right)$, then $\kappa\left(k_{0}, h^{t}\right)=k_{t+1}$. If $t=-1$, then $\kappa\left(k_{0}, \emptyset\right)=k_{0}$.

A strategy profile $\sigma$ induces after any history $h^{t} \in H\left(k_{0}, t\right)$ a (symmetric) strategy profile $\left.\sigma\right|_{h^{t}} \in \Sigma\left(\kappa\left(k_{0}, h^{t}\right)\right)$. For all $s \geq 0, h^{s-1} \in H\left(\kappa\left(k_{0}, h^{t}\right), s-1\right)$, and $\left(\tau_{k, t}, \tau_{\ell, t}\right) \in[\underline{\tau}, \bar{\tau}]^{2}$,

$$
\begin{aligned}
\left.\sigma_{G}\right|_{h^{t}}(s)\left(h^{s-1}\right):= & \sigma_{G}(t+s)\left(h^{t}, h^{s-1}\right) \\
\left.\sigma_{C}\right|_{h^{t}}(s)\left(h^{s-1}, \tau_{k, t+s}, \tau_{\ell, t+s}\right):= & \sigma_{C}(t+s)\left(h^{t}, h^{s-1}, \tau_{k, t+s}, \tau_{\ell, t+s}\right) .
\end{aligned}
$$

That is, $\left.\sigma\right|_{h^{t}}$ generates in period $s$, after history $h^{s-1}$, the same actions as the strategy $\sigma$ generates in period $t+s$, after the history $h^{t}$ followed by the history $h^{s-1}$. 


\section{Walrasian Equilibria}

In this section we characterize the Walrasian equilibrium of the dynamic economy in which a tax policy is arbitrarily specified. Although in our game the government does not have this commitment power, along the equilibrium path, the households act as if the government had established such a policy. Thus, considering arbitrary policies $\tau=\left\{\left(\tau_{k, t}, \tau_{\ell, t}\right)\right\}_{t=0}^{\infty}$ produces an auxiliary problem that is useful in characterizing the sequential equilibria of $\Gamma\left(k_{0}\right)$. This auxiliary problem allows us to focus entirely on the intertemporal incentives of the household.

Definition: A tax policy $\tau=\left\{\left(\tau_{k, t}, \tau_{\ell, t}\right)\right\}_{t=0}^{\infty}$ is feasible if $\left(\tau_{k, t}, \tau_{\ell, t}\right) \in[\underline{\tau}, \bar{\tau}]^{2}$ for all $t \geq 0$.

Definition: Let $\Gamma_{W}\left(k_{0}, \tau\right)$ denote the dynamic Walrasian economy where each household is endowed with $k_{0}$ in period 0 , and the government tax policy is fixed at $\tau$. A symmetric Walrasian equilibrium for $\Gamma_{W}\left(k_{0}, \tau\right)$ is a sequence $\left\{\left(\left(\ell_{t}, c_{t}, k_{t+1}\right),\left(p_{k, t}, p_{\ell, t}\right)\right)\right\}_{t=0}^{\infty}$ of consumption bundles and price vectors such that

$$
p_{k, t}=f_{k}\left(k_{t}, \ell_{t}\right), \quad p_{\ell, t}=f_{\ell}\left(k_{t}, \ell_{t}\right)
$$

for all $t \geq 0$, and the consumption sequence $\left\{\left(\ell_{t}, c_{t}, k_{t+1}\right)\right\}_{t \geq 0}$ solves the following optimization problem

$$
\begin{aligned}
& (P) \quad \max \sum_{t=0}^{\infty} \beta^{t} u\left(\ell_{t}, c_{t}\right) \\
& \text { s.t. } \quad 0 \leq \ell_{t} \leq 1, \quad 0 \leq c_{t}, \quad 0 \leq k_{t+1}, \quad \text { and } \\
& \\
& c_{t}+k_{t+1}=\left(1-\tau_{k, t}\right) p_{k, t} k_{t}+\left(1-\tau_{\ell, t}\right) p_{\ell, t} \ell_{t}, \quad t \geq 0 .
\end{aligned}
$$

Since the prices are uniquely determined by the marginal productivity of capital and labor, sometimes we will omit them and simply specify a Walrasian equilibrium by the con- 
sumption sequence $\left\{\left(\ell_{t}, c_{t}, k_{t+1}\right)\right\}_{t=0}^{\infty}$.

It appears that for a household to be able to make its optimal choices of consumption and investment in period $t$, it needs to correctly anticipate what the other households will do in all future periods (including $t$ ), because the household needs to anticipate current and future prices for labor, capital, and consumption. The future values of taxes and prices, however, affect current decisions only to the extent that they affect the next period's marginal value of capital

$$
m_{t+1}:=\left(1-\tau_{k, t+1}\right) p_{k, t+1} u_{c}\left(\ell_{t+1}, c_{t+1}\right)
$$

That is, correctly predicting the current prices $\left(p_{k, t}, p_{\ell, t}\right)$ as well as next period's marginal value of capital, $m_{t+1}$ is sufficient for the household to make optimal period $t$ decisions. Given capital $k_{t}$, and predictions for $\left(p_{k, t}, p_{\ell, t}\right)$ and $m_{t+1}$, a household's time $t$ problem over $\left(\ell_{t}, c_{t}, k_{t+1}\right)$ can be stated as

$$
\begin{aligned}
& \left(P_{t}\right) \quad \max \quad u\left(\ell_{t}, c_{t}\right)+\beta m_{t+1} k_{t+1} \\
& \text { s.t. } \quad 0 \leq \ell_{t} \leq 1, \quad 0 \leq c_{t}, \quad 0 \leq k_{t+1}, \quad \text { and } \\
& \\
& c_{t}+k_{t+1}=\left(1-\tau_{k, t}\right) p_{k, t} k_{t}+\left(1-\tau_{\ell, t}\right) p_{\ell, t} \ell_{t} .
\end{aligned}
$$

In $\left(P_{t}\right)$, the variable $c_{t}$ can be eliminated using the budget constraint. Then, problem $\left(P_{t}\right)$ can be rewritten as follows:

$$
\begin{aligned}
& \left(P_{t}\right) \quad \max \quad u\left(\ell_{t},\left(1-\tau_{k, t}\right) p_{k, t} k_{t}+\left(1-\tau_{\ell, t}\right) p_{\ell, t} \ell_{t}-k_{t+1}\right)+\beta m_{t+1} k_{t+1} \\
& \text { s.t. } \quad 0 \leq \ell_{t} \leq 1, \quad \text { and } \\
& 0 \leq k_{t+1} \leq\left(1-\tau_{k, t}\right) p_{k, t} k_{t}+\left(1-\tau_{\ell, t}\right) p_{\ell, t} \ell_{t} .
\end{aligned}
$$


Denote by $F\left(k_{t}, \tau_{k, t}, \tau_{\ell, t}, m_{t+1}, p_{k, t}, p_{\ell, t}\right)$ the set of all feasible pairs $\left(\ell_{t}, k_{t+1}\right)$ for problem $\left(P_{t}\right)$ (with $c_{t}$ eliminated). ${ }^{6}$ Clearly, $F\left(k_{t}, \tau_{k, t}, \tau_{\ell, t}, m_{t+1}, p_{k, t}, p_{\ell, t}\right)$ is always a compact, convex polyhedron with nonempty interior (by (A1) and (A3)). Since $u$ is concave, problem $\left(P_{t}\right)$ is convex, and a necessary and sufficient condition for $\left(\ell_{t}, k_{t+1}\right)$ to be optimal is that

$$
(F O C)\left[u_{\ell}\left(\ell_{t}, c_{t}\right)+u_{c}\left(\ell_{t}, c_{t}\right)\left(1-\tau_{\ell, t}\right) p_{\ell, t}\right]\left(\ell-\ell_{t}\right)+\left[-u_{c}\left(\ell_{t}, c_{t}\right)+\beta m_{t+1}\right]\left(k-k_{t+1}\right) \leq 0
$$

for all feasible $(\ell, k) \in F\left(k_{t}, \tau_{k, t}, \tau_{\ell, t}, m_{t+1}, p_{k, t}, p_{\ell, t}\right)$, where

$$
c_{t}:=\left(1-\tau_{k, t}\right) p_{k, t} k_{t}+\left(1-\tau_{\ell, t}\right) p_{\ell, t} \ell_{t}-k_{t+1} .
$$

Definition: Let $\Gamma_{W}^{S}\left(k_{t}, \tau_{k, t}, \tau_{\ell, t}, m_{t+1}\right)$ be the static (one-period) economy where each household has an initial capital stock $k_{t}$ and the government imposes taxes $\left(\tau_{k, t}, \tau_{\ell, t}\right)$. The household chooses consumption bundles $\left(\ell_{t}, c_{t}, k_{t+1}\right)$ and receives utility $u\left(\ell_{t}, c_{t}\right)+\beta m_{t+1} k_{t+1}$ (that is, the utility function is linear in the end-of-period capital stock $k_{t+1}$ ). The vector $\left(\ell_{t}, c_{t}, k_{t+1}, p_{k, t}, p_{\ell, t}\right)$ is a (static) equilibrium for $\Gamma_{W}^{S}\left(k_{t}, \tau_{k, t}, \tau_{\ell, t}, m_{t+1}\right)$ if

$$
\begin{aligned}
& p_{k, t}=f_{k}\left(k_{t}, \ell_{t}\right), \quad p_{\ell, t}=f_{\ell}\left(k_{t}, \ell_{t}\right), \quad \text { and } \\
& \left(c_{t}, \ell_{t}, k_{t+1}\right) \text { solves problem }\left(P_{t}\right) .
\end{aligned}
$$

Let $W E\left(k_{t}, \tau_{k, t}, \tau_{\ell, t}, m_{t+1}\right)$ denote the collection of all Walrasian equilibria of the static economy $\Gamma_{W}^{S}\left(k_{t}, \tau_{k, t}, \tau_{\ell, t}, m_{t+1}\right)$.

It is immediate to see that $\left\{\left(\left(\ell_{t}, c_{t}, k_{t+1}\right),\left(p_{k, t}, p_{\ell, t}\right)\right)\right\}_{t=0}^{\infty}$ is a Walrasian equilibrium of

\footnotetext{
${ }^{6}$ With abuse of notation, sometimes we will say that $\left(\ell_{t}, c_{t}, k_{t+1}\right)$ is in $F\left(k_{t}, \tau_{k, t}, \tau_{\ell, t}, m_{t+1}, p_{k, t}, p_{\ell, t}\right)$ if $\left(\ell_{t}, k_{t+1}\right) \in F\left(k_{t}, \tau_{k, t}, \tau_{\ell, t}, m_{t+1}, p_{k, t}, p_{\ell, t}\right)$ and $c_{t}=\left(1-\tau_{k, t}\right) p_{k, t} k_{t}+\left(1-\tau_{\ell, t}\right) p_{\ell, t} \ell_{t}-k_{t+1}$.
} 
$\Gamma_{W}\left(k_{0}, \tau\right)$ only if for each $t \geq 0$,

$$
\begin{aligned}
& m_{t+1}=\left(1-\tau_{k, t+1}\right) p_{k, t+1} u_{c}\left(\ell_{t+1}, c_{t+1}\right) \quad \text { and } \\
& \left(\ell_{t}, c_{t}, k_{t+1}, p_{k, t}, p_{\ell, t}\right) \in W E\left(k_{t}, \tau_{k, t}, \tau_{\ell, t}, m_{t+1}\right) .
\end{aligned}
$$

The optimality conditions for a static equilibrium are exactly the Euler conditions for problem $(P)$. The next three lemmas establish the converse.

The argument requires us to find a uniform upper bound on the marginal value of capital at any period for all Walrasian equilibria. This upper bound allows us to show that a certain transversality condition is satisfied. To construct such a bound, we first need to find a lower bound $\underline{k}>0$ such that if $\tau$ is any feasible tax policy and $k_{0} \geq \underline{k}$, then the capital stock trajectory of any Walrasian equilibrium of $\Gamma_{W}\left(k_{0}, \tau\right)$ is bounded below by $\underline{k}$.

Lemma 2. There exists $\underline{k}>0$ such that for all $k_{0} \in[\underline{k}, \bar{k}]$ and feasible tax policy $\tau$, each Walrasian equilibrium $\left\{\left(\ell_{t}, c_{t}, k_{t+1}\right)\right\}_{t=0}^{\infty}$ of $\Gamma_{W}\left(k_{0}, \tau\right)$ satisfies $k_{t+1} \geq \underline{k}$ for all $t \geq 0$.

The proof of Lemma 2 is deferred to the Appendix. In what follows, we will only consider initial endowments above the minimum capital associated with Walrasian equilibria, and below the maximal sustainable capital:

(A4) $k_{0} \in[\underline{k}, \bar{k}]$.

Lemma 3. There exists $\overline{\bar{m}}<\infty$ such that for all $k_{0} \in[\underline{k}, \bar{k}]$ and feasible tax policy $\tau=$ $\left\{\left(\tau_{k, t}, \tau_{\ell, t}\right)\right\}_{t=0}^{\infty}$, every Walrasian equilibrium $\left\{\left(\ell_{t}, c_{t}, k_{t+1}\right)\right\}_{t=0}^{\infty}$ of $\Gamma_{W}\left(k_{0}, \tau\right)$ satisfies

$$
m_{t}=\left(1-\tau_{k, t}\right) f_{k}\left(k_{t}, \ell_{t}\right) u_{c}\left(\ell_{t}, c_{t}\right) \leq \overline{\bar{m}} \text { for all } t \geq 0 \text {. }
$$


Proof. Since $f(k, \ell)=f_{k}(k, \ell) k+f_{\ell}(k, \ell) \ell$,

$$
f_{k}(k, \ell) \leq \frac{f(k, \ell)}{k} \leq \frac{f(\bar{k}, 1)}{\underline{k}}=\bar{k} / \underline{k} .
$$

By assumption (A2), $u_{c}\left(\ell_{t}, c_{t}\right) \leq U_{c}$. Hence, we can choose

$$
\overline{\bar{m}}:=(1-\underline{\tau})[\bar{k} / \underline{k}] U_{c}
$$

Lemma 4. Given a feasible tax policy $\tau=\left\{\left(\tau_{k, t}, \tau_{\ell, t}\right)\right\}_{t=0}^{\infty}$ and an initial capital stock $K_{0} \in$ $[\underline{k}, \bar{k}]$, suppose that the sequence $\left\{\left(\left(L_{t}, C_{t}, K_{t+1}\right),\left(p_{k, t}, p_{\ell, t}\right)\right)\right\}_{t=0}^{\infty}$ is such that for each $t$, $\left(L_{t}, C_{t}, K_{t+1}, p_{k, t}, p_{\ell, t}\right) \in W E\left(k_{t}, \tau_{k, t}, \tau_{\ell, t}, m_{t+1}\right)$, where

$$
m_{t+1}:=\left(1-\tau_{k, t+1}\right) f_{k}\left(K_{t+1}, L_{t+1}\right) u_{c}\left(L_{t+1}, C_{t+1}\right) .
$$

Then $\left\{\left(L_{t}, C_{t}, K_{t+1}\right),\left(p_{k, t}, p_{\ell, t}\right)\right\}_{t=0}^{\infty}$ is a Walrasian equilibrium of $\Gamma_{W}\left(K_{0}, \tau\right)$.

Proof. All we need to show is that $\left\{\left(L_{t}, C_{t}, K_{t+1}\right)\right\}_{t=0}^{\infty}$ solves the household dynamic consumption problem $(P)$. For this it is sufficient to show that the sequence satisfies the corresponding transversality condition. Let $\left\{\left(\ell_{t}, c_{t}, k_{t+1}\right)\right\}_{t=0}^{\infty}$ be any feasible sequence; that is, $\left(\ell_{t}, c_{t}, k_{t+1}\right) \in F\left(k_{t}, \tau_{k, t}, \tau_{\ell, t}, m_{t+1}, p_{k, t}, p_{\ell, t}\right)$ for each $t \geq 0$. From the necessary and sufficient condition $(F O C)$ for $\left(P_{t}\right)$ we have that

$$
\begin{array}{r}
u_{\ell}\left(L_{t}, C_{t}\right)\left(\ell_{t}-L_{t}\right)+u_{c}\left(L_{t}, C_{t}\right)\left[\left(1-\tau_{\ell, t}\right) p_{\ell, t}\left(\ell_{t}-L_{t}\right)-\left(k_{t+1}-K_{t+1}\right)\right] \\
\leq-\beta m_{t+1}\left(k_{t+1}-K_{t+1}\right),
\end{array}
$$

and if we add $u_{c}\left(L_{t}, C_{t}\right)\left(1-\tau_{k, t}\right) p_{k, t}\left(k_{t}-K_{t}\right)=m_{t}\left(k_{t}-K_{t}\right)$ to both sides of this inequality, we obtain

$$
u_{\ell}\left(L_{t}, C_{t}\right)\left(\ell_{t}-L_{t}\right)+u_{c}\left(L_{t}, C_{t}\right)\left(c_{t}-C_{t}\right) \leq m_{t}\left(k_{t}-K_{t}\right)-\beta m_{t+1}\left(k_{t+1}-K_{t+1}\right) .
$$


Also, the concavity of $u$ implies that

$$
u\left(\ell_{t}, c_{t}\right) \leq u\left(L_{t}, C_{t}\right)+u_{\ell}\left(L_{t}, C_{t}\right)\left(\ell_{t}-L_{t}\right)+u_{c}\left(L_{t}, C_{t}\right)\left(c_{t}-C_{t}\right) .
$$

Therefore

$$
\begin{aligned}
D & :=\quad \sum_{t=0}^{\infty} \beta^{t}\left[u\left(\ell_{t}, c_{t}\right)-u\left(L_{t}, C_{t}\right)\right] \leq \lim _{T \rightarrow \infty} \sum_{t=0}^{T} \beta^{t}\left[m_{t}\left(k_{t}-K_{t}\right)-\beta m_{t+1}\left(k_{t+1}-K_{t+1}\right)\right] \\
& =\quad \lim _{T \rightarrow \infty} \beta^{T+1} m_{T+1}\left(K_{T+1}-k_{T+1}\right) \leq \lim _{T \rightarrow \infty} \beta^{T+1} m_{T+1} K_{T+1},
\end{aligned}
$$

because $k_{0}=K_{0}$, and $m_{T+1}$ and $k_{T+1}$ are nonnegative. From Lemma 3 we have that $m_{t} \leq \overline{\bar{m}}$ for all $t$. Since $K_{0} \leq \bar{k}$, Lemma 1 implies that $K_{t} \leq \bar{k}$ for all $t$. Therefore, $m_{t} K_{t} \in[0, \overline{\bar{m}} \bar{k}]$ for each $t$, and we have the transversality condition

$$
\lim _{T \rightarrow \infty} \beta^{T} m_{T} K_{T}=0
$$

Hence, $D \leq 0$, and the sequence $\left\{\left(L_{t}, C_{t}, K_{t+1}\right)\right\}_{t=0}^{\infty}$ solves problem $(P)$.

\section{Public Randomization}

We now consider the full game, where the government chooses $\left(\tau_{k, t}, \tau_{\ell, t}\right)$ at the beginning of period $t$. In addition, we now introduce a public randomization device. In every period $t$, before the government makes its choice of tax rates, the government and the households observe the outcome of a random variable $X_{t}$ with a uniform distribution in the interval $[0,1]$. The random variables $\left\{X_{t}\right\}$ are serially uncorrelated and independent of any choices made by the government or the households. The expanded game with public randomization and initial capital stock $k_{0}$ is denoted by $\Gamma^{R}\left(k_{0}\right)$, and $\Gamma^{R}\left(k_{0}, x_{0}\right)$ denotes the subgame of $\Gamma^{R}\left(k_{0}\right)$ that follows after an outcome $x_{0} \in[0,1]$ of the random variable $X_{0}$, before the government makes its move in period 0. Although the random variables are payoff irrelevant, they can 
be used as coordination devices to synchronize the government's and the households' moves and beliefs, in a similar fashion as sunspot equilibria.

We introduce the public randomization device to avoid a certain technical difficulty. Without it, the sets

$$
\left\{\Phi\left(k_{0}, \sigma\right) \mid \sigma \text { is an equilibrium of } \Gamma\left(k_{0}\right)\right\} \subset \mathbf{R}^{2}, \quad k_{0} \in[\underline{k}, \bar{k}],
$$

may fail to be convex. Lemma 5 in Section 5 states that the corresponding sets for the game $\Gamma^{R}\left(k_{0}\right)$ are all convex. Our analysis in Section 5 is facilitated by this property. If the sets above were convex, the public randomization device would be superfluous. Indeed, in that case the corresponding sets for the game with public randomization would coincide with those above.

The history of the game needs to be expanded to record the outcomes of the random devices, and similarly, a strategy must be allowed to react to the random outcomes. The publicly observed history up to the beginning of period $t$ is now $h^{t-1}=\left(h_{0}, \ldots, h_{t-1}\right)$, where

$$
h_{s}:=\left(x_{s}, \tau_{k, s}, \tau_{\ell, s}, \ell_{s}, c_{s}, k_{s+1}\right), \quad s=0, \ldots, t-1
$$

A symmetric strategy profile for $\Gamma^{R}\left(k_{0}\right)$ is a pair of strategies $\sigma=\left(\sigma_{C}, \sigma_{G}\right)$, where for each $t,\left(\tau_{k, t}, \tau_{\ell, t}\right)=\sigma_{G}(t)\left(h^{t-1}, x_{t}\right)$ and $\left(\ell_{t}, c_{t}, k_{t+1}\right)=\sigma_{C}(t)\left(h^{t-1}, x_{t}, \tau_{k, t}, \tau_{\ell, t}\right)$, and both $\sigma_{G}(t)\left(h^{t-1}, x_{t}\right)$ and $\sigma_{C}(t)\left(h^{t-1}, x_{t}, \tau_{k, t}, \tau_{\ell, t}\right)$ are measurable functions of $x_{t}$.

The (stochastic) outcome path $\left\{\left(\tau_{k, t}, \tau_{\ell, t}, \ell_{t}, c_{t}, k_{t+1}\right)\right\}_{t=0}^{\infty}$ generated by a symmetric strategy profile is now a sequence of measurable functions where for each $t$,

$$
\tau_{k, t}, \tau_{\ell, t}, \ell_{t}, c_{t}, k_{t+1}:[0,1]^{t+1} \rightarrow \mathbf{R}_{+}
$$

The actual realizations $\left(\tau_{k, t}\left(x^{t}\right), \tau_{\ell, t}\left(x^{t}\right), \ell_{t}\left(x^{t}\right), c_{t}\left(x^{t}\right), k_{t+1}\left(x^{t}\right)\right)$ in period $t$ are random since they depend on the sequence of random outcomes $x^{t}=\left(x_{0}, \ldots, x_{t}\right)$. 
Finally, we need to distinguish between the expected marginal value of capital and expected value of a symmetric strategy before and after the realization of the random outcome $X_{0}$. Let

$$
\begin{aligned}
& \Phi_{C}^{R}\left(k_{0}, x_{0}, \sigma\right):=\left(1-\tau_{k, 0}\left(x_{0}\right)\right) f_{k}\left(k_{0}, \ell_{0}\left(x_{0}\right)\right) u_{c}\left(\ell_{0}\left(x_{0}\right), c_{0}\left(x_{0}\right)\right) \quad \text { and } \\
& \Phi_{G}^{R}\left(k_{0}, x_{0}, \sigma\right):=\quad E\left[(1-\beta) \sum_{t=0}^{\infty} \beta^{t}\left[u\left(\ell_{t}, c_{t}\right)+g\left(\tau_{k, t} p_{k, t} k_{t}+\tau_{\ell, t} p_{\ell, t} \ell_{t}\right)\right] \mid x_{0}\right]
\end{aligned}
$$

be respectively $\sigma$ 's marginal value of capital and expected value, given the outcome $x_{0}$ of $X_{0}$ (the former is deterministic once $x_{0}$ is known since it only depends on time 0 variables). The corresponding expected marginal value of capital and expected value of $\sigma$ at the beginning of $\Gamma^{R}\left(k_{0}\right)$ (before the realization of $x_{0}$ ) are

$$
\Phi_{C}\left(k_{0}, \sigma\right):=\int_{0}^{1} \Phi_{C}^{R}\left(k_{0}, x_{0}, \sigma\right) d x_{0} \quad \text { and } \quad \Phi_{G}\left(k_{0}, \sigma\right):=\int_{0}^{1} \Phi_{G}^{R}\left(k_{0}, x_{0}, \sigma\right) d x_{0} .
$$

Let $\Sigma^{R}\left(k_{0}\right)=\Sigma_{C}^{R}\left(k_{0}\right) \times \Sigma_{G}^{R}\left(k_{0}\right)$ denote the set of symmetric strategies for $\Gamma^{R}\left(k_{0}\right)$, and $\Sigma^{R}\left(k_{0}, x_{0}\right)=\Sigma_{C}^{R}\left(k_{0}, x_{0}\right) \times \Sigma_{G}^{R}\left(k_{0}, x_{0}\right)$ denote the set of symmetric strategies for $\Gamma^{R}\left(k_{0}, x_{0}\right)$.

Now, a feasible tax policy $\tau=\left\{\left(\tau_{k, t}, \tau_{\ell, t}\right)\right\}_{t=0}^{\infty}$ is a sequence of measurable functions $\left(\tau_{k, t}, \tau_{\ell, t}\right):[0,1]^{t+1} \rightarrow[\underline{\tau}, \bar{\tau}]^{2}, t \geq 0$, that select a pair of tax rates for each sequence of realizations $x^{t}=\left(x_{0}, \ldots, x_{t}\right)$ of the random device. Denote by $\Gamma_{W}^{R}\left(k_{0}, \tau\right)$ the Walrasian economy where each household is initially endowed with $k_{0}$, and the government tax policy is given by $\tau$. A Walrasian equilibrium for $\Gamma_{W}^{R}\left(k_{0}, \tau\right)$ is a sequence of functions $\left\{\left(\left(\ell_{t}, c_{t}, k_{t+1}\right),\left(p_{k, t}, p_{\ell, t}\right)\right)\right\}_{t=0}^{\infty}$ such that

$$
p_{k, t}\left(x^{t}\right)=f_{k}\left(k_{t}\left(x^{t-1}\right), \ell_{t}\left(x^{t}\right)\right), \quad p_{\ell, t}\left(x^{t}\right)=f_{\ell}\left(k_{t}\left(x^{t-1}\right), \ell_{t}\left(x^{t}\right)\right),
$$

for all $x^{t} \in[0,1]^{t+1}$ and $t \geq 0$ (when $t=0, k_{0}$ is not a function), and the random consumption 
sequence $\left\{\left(\ell_{t}, c_{t}, k_{t+1}\right)\right\}$ solves the stochastic optimization problem

$$
\begin{aligned}
& \left(P^{R}\right) \quad \max \quad E\left[\sum_{t=0}^{\infty} \beta^{t} u\left(\ell_{t}\left(x^{t}\right), c_{t}\left(x^{t}\right)\right)\right] \\
& \text { s.t. } \quad 0 \leq \ell_{t}\left(x^{t}\right) \leq 1, \quad 0 \leq c_{t}\left(x^{t}\right), \quad 0 \leq k_{t+1}\left(x^{t}\right), \quad \text { and } \\
& c_{t}\left(x^{t}\right)+k_{t+1}\left(x^{t}\right)=\left(1-\tau_{k, t}\left(x^{t}\right)\right) p_{k, t}\left(x^{t}\right) k_{t}\left(x^{t}\right)+\left(1-\tau_{\ell, t}\left(x^{t}\right)\right) p_{\ell, t}\left(x^{t}\right) \ell_{t}\left(x^{t}\right) \\
& \text { for all } x^{t} \in[0,1]^{t+1} \text { and } t \geq 0,
\end{aligned}
$$

where the expectation is over all sequences $\left\{x_{t}\right\}$. As in the economy without public randomization, prices are uniquely determined by capital and labor inputs, and therefore we will sometimes omit them in the specification of a Walrasian equilibrium.

Given functions $\tau_{k, t}, p_{k, t}, \ell_{t}$, and $c_{t}$ on $[0,1]^{t+1}$ for period $t$, the marginal value of capital after a sequence of random outcomes $x^{t}$ is

$$
m_{t}^{R}\left(x^{t}\right):=\left(1-\tau_{k, t}\left(x^{t}\right)\right) p_{k, t}\left(x^{t}\right) u_{c}\left(\ell_{t}\left(x^{t}\right), c_{t}\left(x^{t}\right)\right)
$$

and the expected marginal value of capital (before $x_{t}$ is known) is

$$
m_{t}\left(x^{t-1}\right):=\int_{0}^{1} m_{t}^{R}\left(x^{t}\right) d x_{t}
$$

Suppose that in period $t$ (almost) all households have initial capital $K_{t}$ and the sequence $x^{t}$ has occurred. If the government fixes the tax rates at $\left(\tau_{k, t}, \tau_{\ell, t}\right)$ and a household has initial capital $k_{t}$ and expectations $\left(p_{k, t}, p_{\ell, t}, m_{t+1}\right)$ (where all the parameters $\left(\tau_{k, t}, \tau_{\ell, t}, p_{k, t}, p_{\ell, t}, m_{t+1}\right)$ depend on $\left.x^{t}\right)$, the household will choose $\left(\ell_{t}\left(x^{t}\right), c_{t}\left(x^{t}\right), k_{t+1}\left(x^{t}\right)\right)$ so as to solve the same problem $\left(P_{t}\right)$ we defined earlier for the game without the random device. Put differently, without public randomization, the parameter $m_{t+1}$ represents the linear payoff to holding capital at the end of the period. With public randomization, the parameter $m_{t+1}$ represents an expected end- 
of-period payoff. But since this payoff is linear, the household faces the same optimization problem $\left(P_{t}\right)$.

Lemmas 2-4 can be easily extended to the model with public randomization. Their proofs follow the same logic as their deterministic counterparts and are omitted.

Lemma $2^{\prime}$. There exists $\underline{k}>0$ such that for all $k_{0} \in[\underline{k}, \bar{k}]$ and feasible tax policy $\tau$, every Walrasian equilibrium $\left\{\left(\ell_{t}, c_{t}, k_{t+1}\right)\right\}_{t=0}^{\infty}$ of $\Gamma_{W}^{R}\left(k_{0}, \tau\right)$ satisfies $k_{t+1}\left(x^{t}\right) \geq \underline{k}$ for all $t \geq 0$ and $x^{t} \in[0,1]^{t+1}$.

Lemma $3^{\prime}$. There exists $\overline{\bar{m}}<\infty$ such that for all $k_{0} \in[\underline{k}, \bar{k}]$ and feasible tax policy $\tau$, every Walrasian equilibrium $\left\{\left(\ell_{t}, c_{t}, k_{t+1}\right)\right\}_{t=0}^{\infty}$ of $\Gamma_{W}^{R}\left(k_{0}, \tau\right)$ satisfies $m_{t}^{R}\left(x^{t}\right) \leq \overline{\bar{m}}$ for all $t \geq 0$ and $x^{t} \in[0,1]^{t+1}$.

Lemma $4^{\prime}$. Let $K_{0} \in[\underline{k}, \bar{k}]$ be an initial capital stock and $\tau=\left\{\left(\tau_{k, t}, \tau_{\ell, t}\right)\right\}_{t=0}^{\infty}$ be a feasible tax policy. Suppose that $\left\{\left(L_{t}, C_{t}, K_{t+1}, p_{k, t}, p_{\ell, t}\right)\right\}_{t=0}^{\infty}$ is such that for each $t \geq 0,\left(L_{t}, C_{t}, K_{t+1}\right)$ : $[0,1]^{t+1} \rightarrow \mathbf{R}_{+}^{3}$, and for all $x^{t} \in[0,1]^{t+1}$,

$$
\begin{aligned}
& p_{k, t}\left(x^{t}\right)=f_{k}\left(K_{t}\left(x^{t-1}\right), L_{t}\left(x^{t}\right)\right), \quad p_{\ell, t}\left(x^{t}\right)=f_{\ell}\left(K_{t}\left(x^{t-1}\right), L_{t}\left(x^{t}\right)\right), \text { and } \\
& \left(L_{t}\left(x^{t}\right), C_{t}\left(x^{t}\right), K_{t+1}\left(x^{t}\right), p_{k, t}\left(x^{t}\right), p_{\ell, t}\left(x^{t}\right)\right) \in W E\left(K_{t}\left(x^{t-1}\right), \tau_{k, t}\left(x^{t}\right), \tau_{\ell, t}\left(x^{t}\right), m_{t+1}\left(x^{t}\right)\right),
\end{aligned}
$$

where

$$
m_{t+1}\left(x^{t}\right)=\int_{0}^{1}\left(1-\tau_{k, t+1}\left(x^{t+1}\right) p_{k, t+1}\left(x^{t+1}\right) u_{c}\left(L_{t+1}\left(x^{t+1}\right), C_{t+1}\left(x^{t+1}\right)\right) d x_{t+1} .\right.
$$

Then, $\left\{\left(L_{t}, C_{t}, K_{t+1}, p_{k, t}, p_{\ell, t}\right)\right\}_{t=0}^{\infty}$ is a Walrasian equilibrium of $\Gamma_{W}^{R}\left(K_{0}, \tau\right)$.

We will use the same symbols defined for the game without public randomization to denote the corresponding entities in the game with public randomization. For example, for any symmetric strategy profile $\sigma$ of $\Gamma^{R}\left(k_{0}\right)$ and history $h^{t},\left.\sigma\right|_{h^{t}}$ denotes the strategy induced 
by $\sigma$ after that history. In addition, if $x_{0} \in[0,1],\left.\sigma\right|_{x_{0}}$ will denote the strategy induced in the proper subgame $\Gamma^{R}\left(k_{0}, x_{0}\right)$.

\section{Self-Generation}

We now provide a formal definition of a sequential equilibrium for our policy game. This is a direct adaptation of the same concept for standard dynamic games, and as stated in the introduction, it is the same concept that Chari and Kehoe (1990) call a sustainable equilibrium. We then introduce our strategic dynamic programming method, which combines the Euler conditions deduced in Lemma $4^{\prime}$ with the self-generation technique of Abreu, Pearce and Stacchetti (1990).

Definition: A symmetric strategy profile $\sigma$ is a symmetric sequential equilibrium (SSE) for $\Gamma^{R}\left(k_{0}\right)$ if for any $t \geq 0$, history $h^{t-1} \in H\left(k_{0}, t-1\right)$, and $x_{t} \in[0,1]$,

(1) $\Phi_{G}^{R}\left(k_{t}, x_{t},\left.\sigma\right|_{h^{t-1}}\right) \geq \Phi_{G}^{R}\left(k_{t}, x_{t},\left(\left.\sigma_{C}\right|_{h^{t-1}}, \gamma\right)\right)$ for all strategies $\gamma \in \Sigma_{G}^{R}\left(k_{t}, x_{t}\right)$ for the government, where $k_{t}=\kappa\left(k_{0}, h^{t-1}\right)$.

(2) If $\left\{\left(\tau_{k, s}^{\prime}, \tau_{\ell, s}^{\prime}, \ell_{s}^{\prime}, c_{s}^{\prime}, k_{s+1}^{\prime}\right)\right\}_{s=0}^{\infty}$ is the stochastic outcome path of $\left.\sigma\right|_{h^{t-1}}$, and $\tau^{\prime}:=\left\{\left(\tau_{k, s}^{\prime}\right.\right.$, $\left.\left.\tau_{\ell, s}^{\prime}\right)\right\}_{s=0}^{\infty}$, then $\left\{\left(\ell_{s}^{\prime}, c_{s}^{\prime}, k_{s+1}^{\prime}\right)\right\}_{s=0}^{\infty}$ is a Walrasian equilibrium of $\Gamma_{W}^{R}\left(k_{t}, \tau^{\prime}\right)$.

Although we have not defined an SSE for $\Gamma^{R}\left(k_{0}, x_{0}\right)$ explicitly, since $\Gamma^{R}\left(k_{0}, x_{0}\right)$ is a proper subgame of $\Gamma^{R}\left(k_{0}\right)$, the following characterization can be used as a "backward" definition: $\sigma$ is an SSE of $\Gamma^{R}\left(k_{0}\right)$ iff $\left.\sigma\right|_{x_{0}}$ is an $\operatorname{SSE}$ of $\Gamma^{R}\left(k_{0}, x_{0}\right)$ for each $x_{0} \in[0,1]$.

Let $h^{t-1}$ be an arbitrary history (not necessarily on the equilibrium path), $k_{t}=$ $\kappa\left(k_{0}, h^{t-1}\right)$, and $\tau^{\prime}$ be the tax policy expected in equilibrium from that point on. Condition (1) of an SSE guarantees that the government will not wish to change $\tau^{\prime}$. Condition (2) 
requires that after $h^{t-1}$, the households follow a Walrasian equilibrium of $\Gamma_{W}^{R}\left(k_{t}, \tau^{\prime}\right)$. That is, each household that starts with $k_{t}$ follows an optimal consumption-investment trajectory (given $\tau^{\prime}$ and what the other households do). Of course, a household with initial capital $k \neq k_{t}$ may do better than (and will not follow) the Walrasian equilibrium. But this is irrelevant: at the time the deviant household chose a consumption-investment bundle different from that expected in equilibrium, it lowered its total expected utility, and along the equilibrium path (when the government does not change the tax policy and the households follow the corresponding Walrasian equilibrium), the deviant household will never recover the losses.

Definition: The ex-ante and ex-post equilibrium correspondences are the set-valued functions $V:[\underline{k}, \bar{k}] \rightarrow \mathbf{R}^{2}$ and $V^{R}:[\underline{k}, \bar{k}] \times[0,1] \rightarrow \mathbf{R}^{2}$ that to each initial capital per capita $k_{0}$ and outcome $x_{0}$ associate the sets

$$
\begin{aligned}
V\left(k_{0}\right) & :=\left\{\Phi\left(k_{0}, \sigma\right) \mid \sigma \text { is an SSE for } \Gamma^{R}\left(k_{0}\right)\right\} \\
V^{R}\left(k_{0}, x_{0}\right) & :=\left\{\Phi^{R}\left(k_{0}, x_{0}, \sigma\right) \mid \sigma \text { is an SSE for } \Gamma^{R}\left(k_{0}, x_{0}\right)\right\} .
\end{aligned}
$$

That is, $V\left(k_{0}\right)$ is the collection of all the pairs $(m, v)$ for which there exists an SSE $\sigma$ such that $m$ is its expected marginal value of investment for consumers in the first period and $v$ is its corresponding expected value for the government.

Lemma 5. For every $k_{0}$ and $x_{0}, x_{0}^{\prime} \in[0,1], V^{R}\left(k_{0}, x_{0}\right)=V^{R}\left(k_{0}, x_{0}^{\prime}\right)$ and $V\left(k_{0}\right)=$ co $\left(V^{R}\left(k_{0}, x_{0}\right)\right)$, where for any subset $X$ in a Euclidean space, $\operatorname{co}(X)$ denotes the convex hull of $X$.

Proof. For every $k_{0}$ and $x_{0}, x_{0}^{\prime} \in[0,1], V^{R}\left(k_{0}, x_{0}\right)=V^{R}\left(k_{0}, x_{0}^{\prime}\right)$ because the subgames 
$\Gamma^{R}\left(k_{0}, x_{0}\right)$ and $\Gamma^{R}\left(k_{0}, x_{0}^{\prime}\right)$ are identical. For every $x_{0} \in[0,1]$, an SSE $\sigma$ of $\Gamma^{R}\left(k_{0}\right)$ selects a continuation SSE $\left.\sigma\right|_{x_{0}} \in \Gamma^{R}\left(k_{0}, x_{0}\right)$ with value $v_{0}^{R}\left(x_{0}\right)$ and marginal value of capital $m_{0}^{R}\left(x_{0}\right)$. That is, $\left(m_{0}^{R}\left(x_{0}\right), v_{0}^{R}\left(x_{0}\right)\right) \in V^{R}\left(k_{0}, x_{0}\right)$. Before the random outcome $x_{0}$ is known, the value of $\sigma$ for the government is the expected value of $v_{0}^{R}\left(x_{0}\right)$, and its marginal value of capital is the expected value of $m_{0}^{R}\left(x_{0}\right)$. For any positive weights $\alpha_{i}, i=1,2,3$ that add up to 1 , and any three points $\left(m_{i}, v_{i}\right) \in V^{R}\left(k, x_{0}\right)$, partition $[0,1]$ into three intervals $I_{i}, i=1,2,3$, so that $\lambda\left(I_{i}\right)=\alpha_{i}$ (here $\lambda$ denotes the Lebesgue measure). For each $x_{0} \in I_{i}$ choose $\left(m_{0}^{R}\left(x_{0}\right), v_{0}^{R}\left(x_{0}\right)\right)=\left(m_{i}, v_{i}\right)$, $i=1,2,3$. Then, $\Phi\left(k_{0}, \sigma\right)=\sum \alpha_{i}\left(m_{i}, v_{i}\right)$, and by Caratheodory's theorem, we have $V\left(k_{0}\right)=$ $\operatorname{co}\left(V^{R}\left(k_{0}, x_{0}\right)\right)$.

Thus, $V\left(k_{0}\right)$ is convex for each $k_{0}$. We also show below that $V\left(k_{0}\right)$ is compact-valued, and so, as we try to characterize the correspondence $V$, we restrict attention to value correspondences.

Definition: An arbitrary correspondence $W:[\underline{k}, \bar{k}] \rightarrow \mathbf{R}^{2}$ with compact and convex values is called a value correspondence. The graph of a value correspondence $W$ is the set

$$
\operatorname{graph}(W):=\{(k,(m, v)) \mid(m, v) \in W(k), k \in[\underline{k}, \bar{k}]\} .
$$

Let $\overline{\mathbf{R}}:=\mathbf{R} \cup\{+\infty,-\infty\}$. For any value correspondence $W$, there exist two functions $\underline{W}, \bar{W}:[\underline{k}, \bar{k}] \times \mathbf{R} \rightarrow \overline{\mathbf{R}}$ such that for each $k \in[\underline{k}, \bar{k}],(m, v) \in W(k)$ iff $\underline{W}(k, m) \leq v \leq$ $\bar{W}(k, m)$. Moreover, $\bar{W}(k, m)$ is concave in $m$ and $\underline{W}(k, m)$ is convex in $m$. If for a given $k$ and $m$ there doesn't exist $v$ such that $(m, v) \in W(k)$, then $\underline{W}(k, m):=+\infty$ and $\bar{W}(k, m):=$ $-\infty$. The graphs of the functions $\underline{W}(k, \cdot)$ and $\bar{W}(k, \cdot)$ trace respectively the lower and upper boundary of $W(k)$. Let $\left[\underline{m}_{W}(k), \bar{m}_{W}(k)\right]$ and $\left[\underline{v}_{W}(k), \bar{v}_{W}(k)\right]$ be respectively the projections 
of $W(k)$ into the first and second coordinates. That is,

$$
\begin{aligned}
\underline{m}_{W}(k):= & \min \{m \mid(m, v) \in W(k)\} \\
\bar{m}_{W}(k):= & \max \{m \mid(m, v) \in W(k)\} \\
\underline{v}_{W}(k):= & \min \{v \mid(m, v) \in W(k)\} \\
\bar{v}_{W}(k):= & \max \{v \mid(m, v) \in W(k)\} .
\end{aligned}
$$

We now turn to decomposing our dynamic game into a series of static games. Each static game corresponds to the strategic problem between the government and the households in a period (with appropriate expectations and modified payoffs).

Fix $x_{0} \in[0,1]$. Suppose that $\sigma$ is an $\operatorname{SSE}$ of $\Gamma^{R}(k)$ (so that $\left.\sigma\right|_{x_{0}}$ is an $\operatorname{SSE}$ of $\Gamma^{R}\left(k, x_{0}\right)$ ) which specifies that after $x_{0}$ in period 0 the government chooses $\left(\tau_{k}, \tau_{\ell}\right)$ and each household chooses $\left(\ell, c, k_{+}\right)$(if the government chooses $\left.\left(\tau_{k}, \tau_{\ell}\right)\right)$. In addition, let $h_{0}:=\left(x_{0}, \tau_{k}, \tau_{\ell}, \ell, c, k_{+}\right)$ and $\left(m_{+}, v_{+}\right)=\Phi\left(k_{+},\left.\sigma\right|_{h^{0}}\right)$ denote the expected marginal value of capital and expected value of the continuation strategy $\left.\sigma\right|_{h^{0}} \in \Sigma^{R}\left(k_{+}\right)$. Since $\sigma$ is an $\operatorname{SSE}$ of $\Gamma^{R}(k),\left.\sigma\right|_{h^{0}}$ is an SSE of $\Gamma^{R}\left(k_{+}\right)$, and therefore $\left(m_{+}, v_{+}\right) \in V\left(k_{+}\right)$. Obviously, the SSE $\sigma$ also specifies what happens when the government adopts an unexpected tax policy for period 0 (that is, a tax policy $\left.\left(\tau_{k}^{\prime}, \tau_{\ell}^{\prime}\right) \neq\left(\tau_{k}, \tau_{\ell}\right)\right)$. This might involve a continuation strategy especially tailored to punish each possible deviation by the government in period 0. However, as in Abreu's (1986, 1988) optimal punishment for firms in a cartel, without loss of generality we consider here extreme punishments only. The incentive constraints that the $\operatorname{SSE} \sigma$ must satisfy in period 0 imply several conditions on $h_{0}$. When the value correspondence $W$ is the equilibrium value correspondence $V$, the following definition captures all the conditions for $h_{0}$ to be the period 
0 outcome of an SSE $\sigma \in \Sigma^{R}\left(k, x_{0}\right)$ and for $\left(m_{+}, v_{+}\right)$to be the expected marginal utility of investment and expected value $\left(\Phi_{C}\left(k,\left.\sigma\right|_{h^{0}}\right), \Phi_{G}\left(k,\left.\sigma\right|_{h^{0}}\right)\right)$ of the corresponding continuation strategy $\left.\sigma\right|_{h^{0}}$.

Definition: A tuple $\xi=\left(\tau_{k}, \tau_{\ell}, \ell, c, k_{+}, m_{+}, v_{+}\right)$is consistent with respect to the value correspondence $W$ at endowment $k \in[\underline{k}, \bar{k}]$ if

(1) $\left(\ell, c, k_{+}, f_{k}(k, \ell), f_{\ell}(k, \ell)\right) \in W E\left(k, \tau_{k}, \tau_{\ell}, m_{+}\right)$, and

(2) $k_{+} \in[\underline{k}, \bar{k}]$ and $\left(m_{+}, v_{+}\right) \in W\left(k_{+}\right)$.

The tuple's marginal value of capital is

$$
\Psi_{C}(k, \xi):=\left(1-\tau_{k}\right) f_{k}(k, \ell) u_{c}(\ell, c)
$$

and its corresponding value is ${ }^{7}$

$$
\Psi_{G}(k, \xi):=(1-\beta)\left[u(\ell, c)+g\left(\tau_{k} f_{k}(k, \ell) k+\tau_{\ell} f_{\ell}(k, \ell) \ell\right)\right]+\beta v_{+} .
$$

For each $\left(\tau_{k}^{\prime}, \tau_{\ell}^{\prime}\right) \in[\underline{\tau}, \bar{\tau}]^{2}$, let

$$
\begin{array}{cc}
\pi_{W}\left(k, \tau_{k}^{\prime}, \tau_{\ell}^{\prime}\right):= & \\
\min _{\left(\ell^{\prime}, c^{\prime}, k_{+}^{\prime}, m_{+}^{\prime}\right)} & (1-\beta) u\left(c^{\prime}, \ell^{\prime}\right)+g\left(\tau_{k}^{\prime} f_{k}\left(k, \ell^{\prime}\right) k+\tau_{\ell}^{\prime} f_{\ell}\left(k, \ell^{\prime}\right) \ell^{\prime}\right)+\beta \underline{W}\left(k_{+}^{\prime}, m_{+}^{\prime}\right) \\
\text { s.t. } & \left(\ell^{\prime}, c^{\prime}, k_{+}^{\prime}\right) \in W E\left(k, \tau_{k}^{\prime}, \tau_{\ell}^{\prime}, m_{+}^{\prime}\right) .
\end{array}
$$

Denote by $M^{*}\left(k, \tau_{k}^{\prime}, \tau_{\ell}^{\prime}\right)$ an optimal choice for $m_{+}^{\prime}$ and by $W E^{*}\left(k, \tau_{k}^{\prime}, \tau_{\ell}^{\prime}\right)$ a corresponding optimal choice for $\left(\ell^{\prime}, c^{\prime}, k_{+}^{\prime}\right)$. That is, $\left(\ell^{\prime}, c^{\prime}, k_{+}^{\prime}, m_{+}^{\prime}\right)=\left(W E^{*}\left(k, \tau_{k}^{\prime}, \tau_{\ell}^{\prime}\right), M^{*}\left(k, \tau_{k}^{\prime}, \tau_{\ell}^{\prime}\right)\right)$ is an

\footnotetext{
${ }^{7}$ For a strategy profile $\sigma$, we previously defined its value $\Phi_{G}(\sigma)$ and marginal value of capital $\Phi_{C}(\sigma)$. Although $\Phi$ and $\Psi$ are similar functions, they are defined on different domains.
} 
optimal solution for the problem above. Define

$$
\begin{array}{r}
\bar{\pi}_{W}(k)=\max \pi_{W}\left(k, \tau_{k}^{\prime}, \tau_{\ell}^{\prime}\right) \\
\text { s.t. }\left(\tau_{k}^{\prime}, \tau_{\ell}^{\prime}\right) \in[\underline{\tau}, \bar{\tau}]^{2} .
\end{array}
$$

The tuple $\xi$ is admissible with respect to the value correspondence $W$ at endowment $k$ if it is consistent with $W$ at $k$ and

(3) $\Psi_{G}(k, \xi) \geq \bar{\pi}_{W}(k)$.

Condition (1) states that given the households' beliefs regarding tomorrow's marginal value of capital $\left(m_{+}\right)$and the current tax rates established by the government, the households respond optimally. Condition (2) requires that the continuation value $v_{+}$together with the marginal utility of investment $m_{+}$can be delivered by an SSE of the next period's subgame $\Gamma^{R}\left(k_{+}\right)$, in which the households start with the amount of capital $k_{+}$they invest this period. Condition (3) of admissibility is the government's incentive constraint. When the government announces unexpected tax rates $\left(\tau_{k}^{\prime}, \tau_{\ell}^{\prime}\right)$, the households' beliefs are manipulated in the ensuing subgame so as to yield the worst possible payoff for the government. Suppose this entails the belief that the marginal utility of investment will be $m_{+}^{\prime}$, and that given this belief, each household saves $k_{+}^{\prime}$ for production next period. Note that the choice of continuation value $v_{+}^{\prime}$ for next period does not affect the optimization problem of the households this period (in particular, it does not affect the choice $k_{+}^{\prime}$ of capital investment). Therefore, the worst punishment must choose the continuation value $v_{+}^{\prime}=\underline{W}\left(k_{+}^{\prime}, m_{+}^{\prime}\right) .8$

\footnotetext{
${ }^{8}$ The only reason to require in the definition of admissibility that the correspondence $W$ be compact-valued is to guarantee that in condition $(3), \underline{W}\left(k_{+}^{\prime}, m_{+}^{\prime}\right)$ is attained. We could define admissibility for an arbitrary
} 
If $W_{1}$ and $W_{2}$ are two value correspondences, we write $W_{1} \subset W_{2}$ if $\operatorname{graph}\left(W_{1}\right) \subset$ $\operatorname{graph}\left(W_{2}\right)$, that is, if $W_{1}(k) \subset W_{2}(k)$ for all $k \in[\underline{k}, \bar{k}]$.

Definition: For any value correspondence $W$, let $B(W)$ denote the correspondence defined by $B(W)(k)=\operatorname{co}\left(B^{R}(W)(k)\right)$ for each $k$, where

$$
B^{R}(W)(k):=\left\{\Psi(k, \xi) \mid \xi=\left(\tau_{k}, \tau_{\ell}, c, \ell, k_{+}, m_{+}, v_{+}\right) \text {is admissible w.r.t. } W \text { at } k\right\} .
$$

Further, a value correspondence $W$ is self-generating if $W \subset B(W)$.

Put simply, $B(W)(k)$ is the convex hull of all points $(m, v)$ for which there exists an admissible tuple w.r.t. $W$ at $k$ with marginal value of capital $m$ and value $v$. More formally, let $k \in[\underline{k}, \bar{k}]$ and $(m, v) \in B(W)(k)$. Since $B(W)(k)=\operatorname{co}\left(B^{R}(W)(k)\right)$, there exist functions $\tau_{k}, \tau_{\ell}, \ell, c, k_{+}, m_{+}, v_{+}:[0,1] \rightarrow \mathbf{R}$ such that for each $x \in[0,1]$, the tuple $\left(\tau_{k}(x), \tau_{\ell}(x), \ell(x), c(x), k_{+}(x), m_{+}(x), v_{+}(x)\right)$ is admissible w.r.t. $W$ at $k$ and

$$
(m, v)=\int_{0}^{1} \Psi\left(k,\left(\tau_{k}, \tau_{\ell}, \ell, c, k_{+}, m_{+}, v_{+}\right)(x)\right) d x .
$$

Moreover, the functions can be chosen to be measurable, and so the expected value is well defined (see the proof of Theorem 1 in the Appendix).

For any value correspondence $W$ and any $k$, the computation of $B^{R}(W)(k)$ can be divided into two steps. Let

$$
\hat{B}(W)(k):=\left\{\Psi(k, \xi) \mid \xi=\left(\tau_{k}, \tau_{\ell}, \ell, c, k_{+}, m_{+}, v_{+}\right) \text {is consistent w.r.t. } W \text { at } k\right\} .
$$

correspondence $W$, if we replace the definition of $\underline{W}(k, m)$ by

$$
\inf \{v \mid(m, v) \in W(k)\} .
$$

(Again here, we use the convention that inf $\emptyset=+\infty$.) This observation is used later in the proof of Theorem 3 . 
Then, for all $k$,

$$
B^{R}(W)(k)=\left\{(m, v) \in \hat{B}(W)(k) \mid v \geq \bar{\pi}_{W}(k)\right\} .
$$

Thus, consistency and the government's incentive constraints are "separable": the latter is only required at the end to truncate, for each $k$, the set obtained when the constraint is neglected. Let $W^{\prime}=B(W)$. Then $\underline{v}_{W^{\prime}}(k)=\bar{\pi}_{W}(k)$. Moreover, the set $B(W)(k)$ is often "flat at the bottom," and $\underline{W}^{\prime}(k, m)=\bar{\pi}_{W}(k)$ for all $m \in[\underline{m}(k), \bar{m}(k)]$.

The following theorem is an adaptation of the similar result proven by Abreu, Pearce and Stacchetti $(1986,1990)$ for repeated games. For completeness, its proof is presented in the Appendix.

Theorem 1. (Self-Generation) If the value correspondence $W$ is self-generating, then $B(W) \subset V$.

The next theorem asserts that the map $B$ transforms value correspondences into value correspondences. Its proof, which is also relegated to the Appendix, uses the following properties of an arbitrary correspondence $W$. Let $X \subset \mathbf{R}^{a}$ and $W: X \rightarrow \mathbf{R}^{b}$ be a compact-valued correspondence. If $X$ is compact, then $W$ is upper semicontinuous (usc) iff graph(W) is a closed subset of $\mathbf{R}^{a} \times \mathbf{R}^{b}$ (see the Corollary to Theorem 7, Chapter VI of Berge (1963)). Moreover, if $W$ is usc, the image $W(K)$ of any compact set $K \subset \mathbf{R}^{a}$ is a compact set in $\mathbf{R}^{b}$ (see Theorem 3, Chapter VI of Berge (1963)). Since $[\underline{k}, \bar{k}]$ is compact, these two properties imply that for any value correspondence $W, \operatorname{graph}(W)$ is a compact subset of $\mathbf{R}^{3}$ iff $W$ is usc.

Theorem 2. If $W$ is an upper semicontinuous (usc) value correspondence, then $B(W)$ is a 
usc value correspondence.

Lemma 6. $\operatorname{graph}(V)$ is a bounded set.

Proof. Let $\sigma$ be any SSE of $\Gamma^{R}\left(k_{0}\right)$. The government's income is always nonnegative, and a household can choose not to work and not to save in every period and thus guarantee itself a total payoff of at least $\underline{\underline{v}}:=u(0,0)+g(0)$ in every period. On the other hand, a household cannot get more than $\overline{\bar{v}}:=u(0, \bar{k})+g(\bar{\tau} \bar{k})$ in any period. Hence, $\underline{\underline{v}} \leq \Phi_{G}\left(k_{0}, \sigma\right) \leq \overline{\bar{v}}$.

Now, $\Phi_{C}\left(k_{0}, \sigma\right) \geq 0$ because for each history $h^{t-1}$ and outcome $x_{t}, \tau_{k, t}\left(h^{t-1}, x_{t}\right) \leq \bar{\tau}<$ 1, the marginal productivity of capital is positive, and the marginal utility of consumption is positive. Finally, Lemma 3 (Lemma $3^{\prime}$ ) establishes the bound $\Phi_{C}\left(k_{0}, \sigma\right) \leq \bar{m}$. Therefore, $\operatorname{graph}(V) \subset[\underline{k}, \bar{k}] \times[0, \overline{\bar{m}}] \times[\underline{\underline{v}}, \overline{\bar{v}}]$, and $\operatorname{graph}(V)$ is bounded.

We are now in a position to present our second main result: the equilibrium correspondence $V$ is the largest fixed point of the map $B$. That is, $V=B(V)$, and $W \subset V$ for any self-generating value correspondence $W$.

Theorem 3. (Factorization) The equilibrium correspondence $V$ is the largest fixed point of the map B. Moreover, $V$ is a usc value correspondence.

Proof. Let $\operatorname{cl}(V)$ denote the correspondence whose graph is the closure of $\operatorname{graph}(V)$. Since $\operatorname{graph}(V)$ is bounded, $\operatorname{graph}(\operatorname{cl}(V))$ is compact, and $\operatorname{cl}(V)$ is a usc value correspondence.

Pick any $\operatorname{SSE} \sigma$ of $\Gamma^{R}\left(k_{0}\right)$, and let $(m, v):=\Phi\left(k_{0}, \sigma\right)$. For each $x \in[0,1]$, let $\left(\tau_{k}(x), \tau_{\ell}(x)\right)=\sigma_{G}(0)(x)$ and $\left(\ell(x), c(x), k_{+}(x)\right)=\sigma_{C}(0)\left(x, \tau_{k}(x), \tau_{\ell}(x)\right)$. That is, $h_{0}=$ $\left(x, \tau_{k}(x), \tau_{\ell}(x), \ell(x), c(x), k_{+}(x)\right)$ is the equilibrium outcome in the first period (when the realization of the random public device is $x)$. Also define $\left(m_{+}(x), v_{+}(x)\right)=\Phi\left(k_{+}(x),\left.\sigma\right|_{h_{0}}\right)$. 
Then, the tuple $\xi(x)=\left(\tau_{k}(x), \tau_{\ell}(x), \ell(x), c(x), k_{+}(x), m_{+}(x), v_{+}(x)\right)$ is admissible w.r.t. $V$ at $k_{0}$, and

$$
(m, v)=\int_{0}^{1} \Psi(k, \xi(x)) d x
$$

This implies that $V \subset B(\operatorname{cl}(V)$ ) (we need to use $\operatorname{cl}(V)$ instead of $V$, to make sure that extreme punishment values can be delivered; see footnote 8). By Theorem 2, graph $(B(\operatorname{cl}(V)))$ is compact, so $\operatorname{cl}(V) \subset B(\operatorname{cl}(V))$. That is, $\operatorname{cl}(V)$ is self-generating, and by Theorem 1, we must have that $B(\operatorname{cl}(V)) \subset V$. Therefore, $\operatorname{cl}(V) \subset V$, and $V$ has a closed graph. That is, $V=\operatorname{cl}(V)$. Moreover, the previous inclusions imply that $V \subset B(V) \subset V$, so $V=B(V)$.

The previous theorem establishes that for each $k \in[\underline{k}, \bar{k}]$ and $m$ in the appropriate range, there exists an (worst) equilibrium $\sigma$ for $\Gamma^{R}(k)$ such that $\Phi_{G}(k, \sigma)=\underline{V}(k, m)$, and thus the extreme punishments invoked in the definition of admissibility can be delivered in equilibrium.

We finally extend the algorithm introduced by Abreu, Pearce and Stacchetti (1990) for repeated games, to compute the equilibrium value correspondence. It is easy to see that the maps $B^{R}$ and $B$ are monotone. That is, if $W$ and $W^{\prime}$ are two value correspondences such that $W \subset W^{\prime}$, then $B^{R}(W) \subset B^{R}\left(W^{\prime}\right)$ and $B(W) \subset B\left(W^{\prime}\right)$. Suppose that $W_{0}$ is a value correspondence such that $V \subset W_{0}$ and $W_{0} \supset B\left(W_{0}\right)$. Construct the sequence of value correspondences $\left\{W_{n}\right\}_{n=0}^{\infty}$ inductively as follows:

$$
W_{n+1}=B\left(W_{n}\right) \quad \text { for all } n \geq 0 \text {. }
$$

Then $W_{n} \supset W_{n+1} \supset V$ for all $n \geq 0$. Since the sequence is decreasing (in the sense of set 
inclusion), it has a limit $W_{\infty}$. That is, $W_{\infty}$ is the value correspondence defined by

$$
W_{\infty}(k)=\lim _{n \rightarrow \infty} W_{n}(k)=\bigcap_{n \geq 0} W_{n}(k)
$$

for each $k \in[\underline{k}, \bar{k}]$. By a simple limit argument, we can establish the following result.

THEOREM 4. $W_{\infty}=V$.

We use this algorithm to compute the solution of an example in Section 7. Some of the implementation details are presented in the Appendix.

\section{Best Equilibria and Steady States}

We now study general properties of equilibria. In particular, we focus on best equilibria (given an initial capital stock $k$ and marginal value of capital $m$ ) and on long run behavior. As in Chamley (1986) and Judd (1985), we study the steady state of best equilibria and compare it with the steady state of the optimal policy with commitment. Although our model is simpler than that of Chamley, the steady state of the optimal tax policy (if it exists) exhibits the same properties: the capital tax is 0 . We will see that without commitment and for intermediate values of the discount factor $\beta$, the steady state of an optimal policy does not have this property. Moreover, the steady state delivers an $(m, v)$ pair which is at the bottom of the corresponding $V(k)$ set. That is, if $k$ is the stock in steady state, then $v=\underline{V}(k, m)$.

We first make an observation about admissible tuples $\xi$ at $k$ (alternatively, about SSE's) such that $\Psi(k, \xi)$ is on the upper boundary of $V(k)$.

Suppose that $\xi=\left(\tau_{k}, \tau_{\ell}, \ell, c, k_{+}, m_{+}, v_{+}\right)$is admissible w.r.t. $V$ at $k$. Since the government's incentives are strengthened when its continuation value is increased, it is easy to see that for all $v_{+} \leq \hat{v}_{+} \leq \bar{V}\left(k_{+}, m_{+}\right), \hat{\xi}=\left(\tau_{k}, \tau_{\ell}, \ell, c, k_{+}, m_{+}, \hat{v}_{+}\right)$is also admissible w.r.t. $V$ 
at $k$. It follows that if $v=\Psi_{G}(k, \xi)=\bar{V}(k, m)$, where $m=\Psi_{C}(k, \xi)$, then $v_{+}=\bar{V}\left(k_{+}, m_{+}\right)$. That is, if $(m, v)=\Psi(k, \xi)$ is on the upper boundary of $V(k)$, then $\left(m_{+}, v_{+}\right)$must be on the upper boundary of $V\left(k_{+}\right)$. Equivalently, this last observation can be stated in terms of SSE's. Suppose $\sigma$ is an SSE of $\Gamma^{R}(k)$ such that $\Phi(\sigma)$ is on the upper boundary of $V(k)$. Let $h_{0}=\left(x_{0}, \tau_{k, 0}, \tau_{\ell, 0}, \ell_{0}, c_{0}, k_{1}\right)$ denote the equilibrium outcome of period 0 when the realization of the random device is $x_{0}$. Then, $\Phi\left(k_{1},\left.\sigma\right|_{h_{0}}\right)$ is on the upper boundary of $V\left(k_{1}\right)$. Inductively then, on the equilibrium path, all $\Phi\left(k_{t+1},\left.\sigma\right|_{h_{t}}\right), t \geq 0$, are on the upper boundary of the correspondence $V$ (for all sequences of random outcomes $\left\{x_{t}\right\}_{t=0}^{\infty}$ ).

Definition: A steady state equilibrium for $\Gamma^{R}\left(k^{s}\right)$ is an SSE $\sigma$ whose outcome path $\left\{\left(\tau_{k, t}\right.\right.$, $\left.\left.\tau_{\ell, t}, \ell_{t}, c_{t}, k_{t+1}\right)\right\}_{t=0}^{\infty}$ is stationary. That is, there exist $\left(\tau_{k}^{s}, \tau_{\ell}^{s}, \ell^{s}, c^{s}\right) \in[\underline{\tau}, \bar{\tau}]^{2} \times[0,1] \times[0, \infty)$ such that for all $t \geq 0$ and $x^{t} \in[0,1]^{t+1}$,

$$
\left(\tau_{k, t}, \tau_{\ell, t}, \ell_{t}, c_{t}, k_{t+1}\right)\left(x^{t}\right)=\left(\tau_{k}^{s}, \tau_{\ell}^{s}, \ell^{s}, c^{s}, k^{s}\right)
$$

Note that although the outcome path is not random (i.e., it doesn't depend on the outcomes of the public randomization device), $\sigma$ may use the public randomization device to support "punishments" off the equilibrium path.

Suppose $\sigma$ is a symmetric strategy profile for $\Gamma^{R}\left(k^{*}\right)$ whose outcome path is not random. Let $\left(m^{s}, v^{s}\right)=\Phi\left(k^{*}, \sigma\right)$, and for any $x_{0} \in[0,1]$, let

$$
\left(\tau_{k}^{s}, \tau_{\ell}^{s}\right)=\sigma_{G}(0)\left(x_{0}\right) \quad \text { and } \quad\left(\ell^{s}, c^{s}, k^{s}\right)=\sigma_{C}(0)\left(x_{0}, \tau_{k}^{s}, \tau_{\ell}^{s}\right)
$$

Then, it is easy to see that $\sigma$ is a steady state equilibrium if and only if $\xi^{s}=\left(\tau_{k}^{s}, \tau_{\ell}^{s}, \ell^{s}, c^{s}\right.$, $\left.k^{s}, m^{s}, v^{s}\right)$ is an admissible tuple with respect to $V^{R}$ at $k^{*}$ such that $k^{s}=k^{*}$ and $\left(m^{s}, v^{s}\right)=$ $\Psi\left(k^{*}, \xi^{s}\right)$.

Definition: $\xi^{s}=\left(\tau_{k}^{s}, \tau_{\ell}^{s}, \ell^{s}, c^{s}, k^{s}, m^{s}, v^{s}\right)$ is a steady state tuple if 
(i) $\left(\ell^{s}, c^{s}, k^{s}, f_{k}\left(k^{s}, \ell^{s}\right), f_{\ell}\left(k^{s}, \ell^{s}\right)\right) \in W E\left(k^{s}, \tau_{k}^{s}, \tau_{\ell}^{s}, m^{s}\right)$, and

(ii) $\left(m^{s}, v^{s}\right)=\Psi\left(k^{s}, \xi^{s}\right)$.

Since for a steady state tuple we do not impose admissibility (in particular, we do not impose the government's incentive constraint), steady state tuples are the solutions of a (relatively) simple fixed point problem. If a steady state tuple is not admissible, it cannot be sustained by a steady state equilibrium. However, in the game with full commitment, where the government chooses a tax policy once and for all at the beginning of period 0 , any steady state tuple $\xi^{s}=\left(\tau_{k}^{s}, \tau_{\ell}^{s}, \ell^{s}, c^{s}, k^{s}, m^{s}, v^{s}\right)$ can be supported by a stationary tax policy where $\left(\tau_{k, t}, \tau_{\ell, t}\right)=\left(\tau_{k}^{s}, \tau_{\ell}^{s}\right)$ for all $t \geq 0$. Clearly, since $f(0, \ell)=0$ for all $\ell \geq 0$, there is a trivial steady state tuple where $k^{s}=\ell^{s}=c^{s}=0$.

For the next theorem, we now assume that $\underline{\tau}=0$.

THEOREM 5. Let $\sigma$ be an SSE for $\Gamma^{R}\left(k_{0}\right)$. Suppose that $\Phi_{G}\left(k_{0}, \sigma\right)=\bar{v}_{V}\left(k_{0}\right)$ and that $\sigma$ converges to steady state, represented by the tuple $\xi^{s}=\left(\tau_{k}^{s}, \tau_{\ell}^{s}, \ell^{s}, c^{s}, k^{s}, m^{s}, v^{s}\right)$. Then $\xi^{s}$ is a steady state tuple and either (1) $\tau_{k}^{s}=0$ or (2) $v^{s}=\underline{V}\left(k^{s}, m^{s}\right)=\underline{v}_{V}\left(k^{s}\right)$.

Note that since $\Phi\left(k_{0}, \sigma\right)$ is on the upper boundary of $V\left(k_{0}\right)$, along the outcome path, $\Phi\left(k_{t+1},\left.\sigma\right|_{h_{t}}\right), t \geq 0$, is on the upper boundary of the correspondence $V$. Thus, by continuity, $\left(m^{s}, v^{s}\right)$ is on the upper boundary of $V\left(k^{s}\right)$. That is, $v^{s}=\bar{V}\left(k^{s}, m^{s}\right)$. When $\tau_{k}^{s}>0$, the theorem asserts that $v^{s}=\underline{V}\left(k^{s}, m^{s}\right)$. Therefore, in this case, $\left(m^{s}, v^{s}\right)$ must be one of the two intersection points between the upper and the lower boundaries of $V\left(k^{s}\right)$ (and thus, either $m^{s}=\underline{m}_{V}\left(k^{s}\right)$ or $\left.m^{s}=\bar{m}_{V}\left(k^{s}\right)\right)$. Moreover, the lower boundary must stay (weakly) above the value $\underline{V}\left(k^{s}, m^{s}\right)$. 
Proof. To simplify the notation, we write the proof for the game $\Gamma\left(k_{0}\right)$ without public randomization. Alternatively, assume that the equilibrium value correspondence of the game without public randomization is convex valued (and thus the randomization device is superfluous). For any (deterministic) outcome path $h^{\infty}=\left\{\left(\tau_{k, t}, \tau_{\ell, t}, \ell_{t}, c_{t}, k_{t+1}\right)\right\}_{t=0}^{\infty}$ and any $t \geq 0$, let

$$
S_{t}\left(h^{\infty}\right)=(1-\beta) \sum_{i=0}^{\infty} \beta^{i}\left[u\left(\ell_{t+i}, c_{t+i}\right)+g\left(G_{t+i}\right)\right],
$$

where $G_{t}=\tau_{k, t} f_{k}\left(k_{t}, \ell_{t}\right) k_{t}+\tau_{\ell, t} f_{\ell}\left(k_{t}, \ell_{t}\right) \ell_{t}$. Following Chamley, we can view the government choices of taxes to be equivalent to the choices of after-tax wage $w_{t}$ and "interest rate" on capital $r_{t}$, where

$$
1+r_{t}:=\left(1-\tau_{k, t}\right) f_{k}\left(k_{t}, \ell_{t}\right) \quad \text { and } \quad w_{t}:=\left(1-\tau_{\ell, t}\right) f_{\ell}\left(k_{t}, \ell_{t}\right) \text {. }
$$

We also use the standard notation of $u_{\ell, t}$ and $u_{c, t}$ for $u_{\ell}\left(\ell_{t}, c_{t}\right)$ and $u_{c}\left(\ell_{t}, c_{t}\right)$ respectively. Then (with a small abuse of notation):

$$
\begin{array}{ll}
\Phi_{G}\left(k_{0}, \sigma\right) & =\max S_{0}\left(h^{\infty}\right) \\
\text { s.t } & \left(\ell_{t}, c_{t}, k_{t+1}\right) \in W E\left(k_{t}, r_{t}, w_{t},\left(1+r_{t+1}\right) u_{c, t+1}\right) \\
& c_{t}+k_{t+1}+G_{t} \leq f\left(k_{t}, \ell_{t}\right) \\
& S_{t}\left(h^{\infty}\right) \geq \bar{\pi}_{V}\left(k_{t}\right) .
\end{array}
$$

The first constraint requires that the trajectory of labor, consumption and savings constitutes a Walrasian equilibrium of the dynamic economy that arises when the government fixes its tax policy at $\tau$. The second constraint is the government's budget constraint, and the last is the government's incentive constraint. The latter says that what the government expects in equilibrium from period $t$ onward has to be no less than the worst punishment value for the game $\Gamma\left(k_{t}\right)$. 
Suppose that the solution converges to the steady state represented by the tuple $\xi^{s}=$ $\left(\tau_{k}^{s}, \tau_{\ell}^{s}, \ell^{s}, c^{s}, k^{s}, m^{s}, v^{s}\right)$. Then $k^{s} \geq \underline{k}>0$. It is easy to see that $\ell^{s}>0$, for otherwise $f\left(k^{s}, \ell^{s}\right)=0$, which is incompatible with the government incentive constraint. Since $k^{s}>0$ and $\ell^{s}>0$, we must also have that $c^{s}>0$ (otherwise the consumer could increase his utility by decreasing his savings). If $\ell^{s}<1$ (so that the consumer's choices are "interior"), the Walrasian equilibrium requirement for $t$ sufficiently large is equivalent to the first order conditions

$$
u_{\ell, t}+w_{t} u_{c, t}=0, \quad u_{c, t}=\beta\left(1+r_{t+1}\right) u_{c, t+1}, \quad \text { and } \quad c_{t}+k_{t+1} \leq\left(1+r_{t}\right) k_{t}+w_{t} \ell_{t} .
$$

For simplicity, assume that the consumers' trajectory is "interior" (the alternative case, when $\ell^{s}=1$ can be dealt with in a similar fashion). Then, we can replace the Walrasian equilibrium requirement by its corresponding first-order conditions to obtain

$$
\begin{array}{ll}
\Phi_{G}\left(k_{0}, \sigma\right) & =\max S_{0}\left(h^{\infty}\right) \\
\text { s.t. } & u_{\ell, t}+w_{t} u_{c, t}=0 \\
& u_{c, t}=\beta\left(1+r_{t+1}\right) u_{c, t+1} \\
& c_{t}+k_{t+1} \leq\left(1+r_{t}\right) k_{t}+w_{t} \ell_{t} \\
& c_{t}+k_{t+1}+G_{t} \leq f\left(k_{t}, \ell_{t}\right) \\
& S_{t}\left(h^{\infty}\right) \geq \bar{\pi}_{V}\left(k_{t}\right) .
\end{array}
$$

Let $y_{t} \beta^{t}, z_{t} \beta^{t}, \lambda_{t} \beta^{t}, \mu_{t} \beta^{t}$, and $\gamma_{t} \beta^{t}$ be the Lagrange multipliers corresponding to the five constraints in period $t$. It is easy to see that the third and fourth constraints must be active for all $t \geq 0$. If the government's incentive constraint in period $t$ is not active, then $\gamma_{t}=0$ and the optimality condition associated with $k_{t}$ is

$$
0=\lambda_{t-1}-\lambda_{t} \beta\left(1+r_{t}\right)+\mu_{t-1}-\mu_{t} \beta f_{k, t} .
$$


Assume first that the government's incentive constraint is not active in the limit, that is, that

$$
u\left(\ell^{s}, c^{s}\right)+g\left(G^{s}\right)>\bar{\pi}_{V}\left(k^{s}\right) .
$$

Then, by continuity, $S_{t}\left(h^{\infty}\right)>\bar{\pi}_{V}\left(k_{t}\right)$ for all $t$ sufficiently large. In the limit, the second constraint implies that $\beta\left(1+r^{s}\right)=1$. Therefore, in the limit, the optimality condition for $k_{t}$ implies that $1-\beta f_{k}\left(k^{s}, \ell^{s}\right)=0$. The last two equalities imply that $1+r^{s}=f_{k}\left(k^{s}, \ell^{s}\right)$, or that $\tau_{k}^{s}=0$. This is the Chamley result (and proof). ${ }^{9}$

Recall from Section 5 that for all $k$ and $W, \underline{v}_{B(W)}(k)=\bar{\pi}_{W}(k)$. Since $V=B(V)$, $\bar{\pi}_{V}\left(k^{s}\right)=\underline{v}_{V}\left(k^{s}\right)$. Thus, if the government's incentive constraint is active in the limit, we have

$\lim _{t \rightarrow \infty} \Phi\left(k_{t+1},\left.\sigma\right|_{h^{t}}\right)=\lim _{t \rightarrow \infty} S_{t}\left(h^{\infty}\right)=u\left(\ell^{s}, c^{s}\right)+g\left(G^{s}\right)=\bar{\pi}_{V}\left(k^{s}\right)=\underline{v}\left(k^{s}\right)$.

When the government's incentive constraint is not active in the limit and the Chamley result attains, $\xi^{s}$ must be the best steady state tuple. Whether the best steady state is sustainable by an SSE depends on the discount factor $\beta$ (and on $\bar{\tau}$ as well). In equilibrium, the benefits of a deviation to a higher capital tax and lower labor tax in the first period, for example, are compensated by a lower continuation value for the government. But if the discount factor is low, the former effect dominates, and the government cannot resist the temptation. In this case, the best steady state is not sustainable.

\section{An Example}

In this section we present an example. With the algorithm described at the end of Section 5 (see Theorem 4), which we have implemented on a computer, we find its equilibrium

\footnotetext{
${ }^{9}$ Judd (1985) proves a similar result in a continuous time model.
} 
correspondence. With a similar algorithm, we also find the value correspondence $V^{*}$ of the game with commitment, when the government chooses its tax policy once and for all at the beginning of period 0. Below we compare $V(k)$ with $V^{*}(k)$ for a couple of values of the initial capital stock $k$. We also find the corresponding steady state tuples and identify the Chamley-Judd (or first-best) and second-best steady state outcomes.

In the example we have made the following choices:

$$
\begin{aligned}
& u(\ell, c)=2 \sqrt{c}-\left(1-\ell^{1.1}\right)^{.9}, \quad g(G)=2 \sqrt{G}, \quad \text { and } \quad \beta=0.9 \\
& f(k, \ell)=k^{3} \ell^{\cdot 7}, \quad \text { and } \quad \delta=0.05
\end{aligned}
$$

where $\delta$ represents the capital depreciation rate. As we explained in footnote 3 , we make the assumption of full depreciation (i.e., $\delta=1$ ) throughout the paper for convenience only. Our results also apply to the case $\delta<1 .{ }^{10}$ Finally, to reduce the size of the numerical problem, we assume that values for $\tau_{k}$ and $\tau_{\ell}$ are restricted to be in the discrete grid $\{0,0.1,0.2, \ldots, 0.9\}$. This can be viewed as an institutional constraint, where the government is not allowed other intermediate values of taxes.

The equation $k=(1-\delta) k+f(k, 1)$ gives us $\bar{k}=10 \sqrt{8}$. But this upper bound is too generous. We set instead $\bar{k}=1.57 \times 1.5=2.35 ; k=1.57$ is the largest capital stock that can be sustained in steady state with full commitment (when $\tau_{k, t}=\tau_{\ell, t}=0$ for all $t \geq 0$ ).

\footnotetext{
${ }^{10}$ Since investments are irreversible, the household's problem must now include the constraint $k_{t+1} \geq$ $(1-\delta) k_{t}$ for all $t \geq 0$. The definition of the marginal value of capital is also different and more complex. Recall that $m_{t}$ represents the increase in the household's total utility when the capital stock $k_{t}$ is increased by one unit. Basically, without full depreciation, $m_{t}=u_{c}\left(\ell_{t}, c_{t}\right)\left[\left(1-\tau_{k, t}\right) f_{k}\left(k_{t}, \ell_{t}\right)+(1-\delta)\right]$. This definition is fine as long as capital investment in period $t$ is positive, or $k_{t+1}>(1-\delta) k_{t}$. It assumes that at the end of period $t$, an optimal choice for the household is to invest $(1-\delta)$ less, to return to the original capital stock $k_{t+1}$ for the next period. Thus, at the end of period $t$, the household increases its consumption by $(1-\delta)$ plus the additional income generated by the additional unit of capital stock. The actual definition of $m_{t}$ needs to be more complex to account for the possibility that $k_{t+1}=(1-\delta) k_{t}$.
} 
There does not exist such a direct formula to compute $\underline{k}$. We have taken $\underline{k}=0.034$, which is the ("smallest") capital stock sustained in steady state with full commitment, when $\tau_{k, t}=.9$ and $\tau_{\ell, t}=0$ for all $t \geq 0$. We choose a grid for $k$ with 60 points uniformly spaced between $\underline{k}$ and $\bar{k}$.

Figure 1 (below) displays simultaneously $V(0.744)$ and $V^{*}(0.744)$. Obviously $V(k) \subset$ $V^{*}(k)$ for all $k$. Thus, $V^{*}(0.744)$ is the larger convex set depicted in Figure 1 , and $V(0.744)$ is the smaller set contained in it. The concave curve from $(m, v)=(1.5,2.46)$ to $(m, v)=$ $(2.4,2.45)$ represents the locus of all $\left(m^{s}, v^{s}\right)$ such that $\xi^{s}=\left(\tau_{k}^{s}, \tau_{\ell}^{s}, \ell^{s}, c^{s}, k^{s}, m^{s}, v^{s}\right)$ is a steady state tuple with $k^{s}=0.744$. Figure 2 displays $V^{*}(1.18), V(1.18)$, and the locus of all $\left(m^{s}, v^{s}\right)$ such that $\xi^{s}$ is a steady state tuple with $k^{s}=1.18$. One observation, that holds in all of our simulations, is that the larger is $k$, the smaller $V(k)$ is relative to $V^{*}(k)$.

The value $k=0.744$ is critical: for $k>0.744$, the locus of steady states $\left(m^{s}, v^{s}\right)$ does not intersect $V(k)$. That is, for $k>0.744$ no steady state can be supported by an SSE (this can be seen, for example, in Figure 2, where $k=1.18)$. For $k=0.744$, the locus of $\left(m^{s}, v^{s}\right)$ pairs for steady state tuples is a curve whose leftmost point corresponds to the steady state that attains when $\tau_{k, t}=0.38$ and $\tau_{\ell, t}=0$ for all $t \geq 0$, and the rightmost point corresponds to the steady state that attains when $\tau_{k, t}=0$ and $\tau_{\ell, t}=0.54$ for all $t \geq 0$. The only point of intersection between $V(0.744)$ and the curve represents the second-best steady state and is attained when $\tau_{k, t}=0.29$ and $\tau_{\ell, t}=0.23$ for all $t \geq 0$. This is the best steady state that can be supported by an SSE. As stated in Theorem 5, this intersection coincides with the right corner of $V(0.744)$, where the upper and lower boundaries of $V(0.744)$ intersect. 


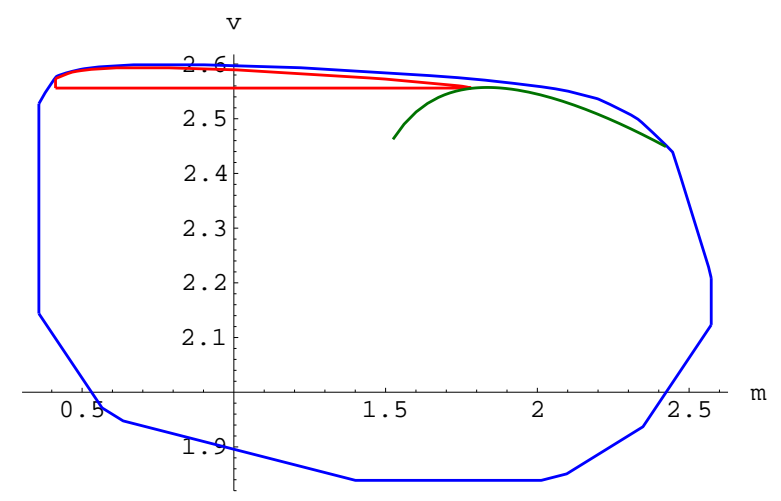

Figure 1: Value Correspondences for $k=.744$

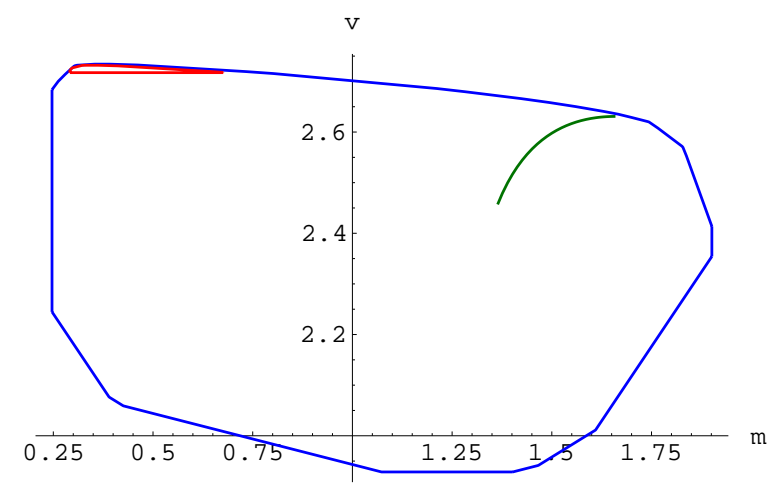

Figure 2: Value Correspondences for $k=1.18$

The first-best steady state is obtained when $k=1.18$. In Figure 2, the locus of steady states $\left(m^{s}, v^{s}\right)$ is an upward sloping curve, whose rightmost point is on the boundary of $V^{*}(1.18)$. The leftmost point of the curve corresponds to the steady state that attains when $\tau_{k, t}=0.17$ and $\tau_{\ell, t}=0$ for all $t \geq 0$, and the rightmost point corresponds to the steady state that attains when $\tau_{k, t}=0$ and $\tau_{\ell, t}=0.28$ for all $t \geq 0$. The latter is the first-best steady state, and in agreement with the Chamley-Judd result, capital taxes are 0. However, as can be seen in Figure 2, none of the steady states for $k=1.18$ can be supported with an SSE. 


\section{Conclusion}

We have elaborated a general method for studying dynamic games with anonymous players. This class of games includes many standard "policy games," where the government chooses a policy (taxes, debt, or inflation) and there is a continuum of agents (households). The method uses the idea that since each household's actions are unobservable and do not affect prices, the household's intertemporal incentives can be captured completely by a single parameter (the marginal value of capital in our model). Although we have developed these ideas in the context of a dynamic game (with the capital stock as state variable), a similar technique would apply to a simpler class of infinitely repeated anonymous games.

The high complexity of these games often makes it impossible to find analytic solutions. However, the extension of the algorithm proposed by Abreu, Pearce and Stacchetti (1990) produces a sequence of approximations to the equilibrium correspondence that converges monotonically, and provides a practical method for constructing the equilibrium correspondence.

\section{Appendix}

Proof of Lemma 2: Let $\tau=\left\{\left(\tau_{k, t}, \tau_{\ell, t}\right)\right\}_{t=0}^{\infty}$ be a feasible tax policy and $\left\{\left(\ell_{t}, c_{t}, k_{t+1}\right)\right\}_{t=0}^{\infty}$ be a Walrasian equilibrium of $\Gamma_{W}\left(k_{0}, \tau\right)$. We will show that there exists $\underline{k}>0$ such that $k_{t+1} \geq \underline{k}$ whenever $k_{t} \geq \underline{k}$, for all $t \geq 0$. Since the proof is for an arbitrary feasible tax policy, it is enough to check that if $k_{0} \geq \underline{k}$, then $k_{1} \geq \underline{k}$.

By contradiction, suppose that there exists a decreasing sequence $\left\{k_{0}^{s}\right\}_{s=0}^{\infty}$ with $\lim _{s \rightarrow \infty} k_{0}^{s}=0$, such that for each $s$ there exist a tax policy $\tau^{s}$ and a Walrasian equilib$\operatorname{rium}\left\{\left(\ell_{t}^{s}, c_{t}^{s}, k_{t+1}^{s}\right)\right\}_{t=0}^{\infty}$ of $\Gamma_{W}\left(k_{0}^{s}, \tau^{s}\right)$ for which $k_{1}^{s}<k_{0}^{s}$. 
Suppose first that $\liminf _{s \rightarrow \infty} \ell_{0}^{s} / k_{0}^{s}<\infty$. Then, there exists a subsequence for which the above labor to capital ratio remains uniformly bounded above, and without loss of generality we can assume that along the subsequence, $\ell_{0}^{s} / k_{0}^{s} \rightarrow r$, for some $r \geq 0$. Along that subsequence, $k_{0}^{s} \rightarrow 0$, which implies that $\ell_{0}^{s} \rightarrow 0$. Since $f_{\ell}$ is homogeneous of degree 0 , we have that

$$
f_{\ell}\left(k_{0}^{s}, \ell_{0}^{s}\right)=f_{\ell}\left(1, \ell_{0}^{s} / k_{0}^{s}\right) \rightarrow f_{\ell}(1, r)>0
$$

Labor taxes are uniformly bounded above by $\bar{\tau}<1$, so after-tax wages in period 0 are bounded below by $(1-\bar{\tau}) f_{\ell}(1, r) / 2>0$, say, for $s$ sufficiently large. But since $k_{0}^{s} \rightarrow 0$ and $\ell_{0}^{s} \rightarrow 0, c_{0}^{s} \leq f\left(k_{0}^{s}, \ell_{0}^{s}\right) \rightarrow 0$. Hence, $u_{\ell}\left(\ell_{0}^{s}, c_{0}^{s}\right) \rightarrow 0$ by assumption (A2). Therefore, for any positive constant $\gamma$, along the subsequence, $-u_{\ell}\left(\ell_{0}^{s}, c_{0}^{s}\right)<\gamma(1-\bar{\tau}) f_{\ell}(1, r) / 2$, for $s$ sufficiently large. Let

$$
\gamma:=\inf _{\ell \in[0,1)} u_{c}(\ell, \bar{k})
$$

which is positive by assumption (A2). (Recall that the budget constraint implies that $\bar{k}=$ $f(\bar{k}, 1)$ is an upper bound on consumption.) Suppose the household works a little harder in period 0 and immediately consumes the additional income. Then its total utility increases by

$$
u_{c}\left(\ell_{0}^{s}, c_{0}^{s}\right)\left(1-\tau_{\ell, 0}^{s}\right) f_{\ell}\left(k_{0}^{s}, \ell_{0}^{s}\right)+u_{\ell}\left(\ell_{0}^{s}, c_{0}^{s}\right)>\gamma(1-\bar{\tau}) f_{\ell}(1, r) / 2+u_{\ell}\left(\ell_{0}^{s}, c_{0}^{s}\right)>0
$$

This is a contradiction. Therefore, we must have that $\lim _{s \rightarrow \infty} \ell_{0}^{s} / k_{0}^{s}=\infty$.

Since $k_{1}^{s}<k_{0}^{s}$ for all $s$, we also have that $k_{1}^{s} \rightarrow 0$, and the same logic as above implies that $\lim _{s \rightarrow \infty} \ell_{1}^{s} / k_{1}^{s}=\infty$. 
By assumption (A3), since $f_{k}$ is homogeneous of degree 0 and $k_{0}^{s} / \ell_{0}^{s} \rightarrow 0$, we have

$$
f_{k}\left(k_{0}^{s}, \ell_{0}^{s}\right)=f_{k}\left(k_{0}^{s} / \ell_{0}^{s}, 1\right) \rightarrow \infty
$$

Hence,

$$
\left(1-\tau_{k, 0}^{s}\right) f_{k}\left(k_{0}^{s}, \ell_{0}^{s}\right) \geq(1-\bar{\tau}) f_{k}\left(k_{0}^{s}, \ell_{0}^{s}\right) \geq 2
$$

for all $s$ sufficiently large, and $k_{1}^{s}<k_{0}^{s}$ implies that

$$
c_{0}^{s} \geq\left(1-\tau_{k, 0}^{s}\right) f_{k}\left(k_{0}^{s}, \ell_{0}^{s}\right) k_{0}^{s}-k_{1}^{s} \geq k_{0}^{s}>0
$$

Since $k_{1}^{s} / \ell_{1}^{s} \rightarrow 0$ as well, we also have

$$
f_{k}\left(k_{1}^{s}, \ell_{1}^{s}\right)=f_{k}\left(k_{1}^{s} / \ell_{1}^{s}, 1\right) \rightarrow \infty
$$

Finally, $u_{c}\left(\ell_{1}^{s}, c_{1}^{s}\right) \geq \gamma>0$, and by assumption $(\mathrm{A} 2), u_{c}\left(\ell_{0}^{s}, c_{0}^{s}\right) \leq U_{c}$. This is a contradiction: for large enough $s$, the household would prefer to save more in period 0 and consume the additional income in period 1 . Since $c_{0}^{s}>0$ the household can save more in period 0 . For each additional dollar it saves, its utility decreases by at most $U_{c}$ in period 0 . However, in period 1 , each additional dollar invested increases the household's utility by at least $(1-\bar{\tau}) f_{k}\left(k_{1}^{s}, \ell_{1}^{s}\right) \gamma$, which tends to $\infty$ as $s \rightarrow \infty$.

Proof of Theorem 1: By definition, for each $k \in[\underline{k}, \bar{k}]$ and $(m, v) \in B(W)(k)$, there exist functions $\tau_{k}, \tau_{\ell}, \ell, c, k_{+}, m_{+}, v_{+}:[0,1] \rightarrow \mathbf{R}$ such that for each $x \in[0,1]$, the tuple $\left(\tau_{k}(x), \tau_{\ell}(x), \ell(x), c(x), k_{+}(x), m_{+}(x), v_{+}(x)\right)$ is admissible w.r.t. $W$ at $k$ and

$$
(m, v)=\int_{0}^{1} \Psi\left(k,\left(\tau_{k}, \tau_{\ell}, \ell, c, k_{+}, m_{+}, v_{+}\right)(x)\right) d x
$$


As argued in the footnote below, the functions can be assumed to be measurable, and so the expected value is well defined. ${ }^{11}$ Let $A$ be a map that for each $(k, m, v) \in \operatorname{graph}(B(W))$ selects a function $A(k, m, v):[0,1] \rightarrow \mathbf{R}^{7}$ such that for each $x \in[0,1], A(k, m, v)(x)$ is an admissible tuple w.r.t. $W$ at $k$, and

$$
(m, v)=\int_{0}^{1} \Psi(k, A(k, m, v)(x)) d x .
$$

For each $x, A(k, m, v)(x)$ is a 7-dimensional vector, where, for example, its first coordinate $A_{1}(k, m, v)(x)$ represents the capital tax rate $\tau_{k}(x)$ of the tuple.

For each $\left(k_{0}, m_{0}, v_{0}\right) \in \operatorname{graph}(B(W))$ we now construct a symmetric strategy profile $\hat{\sigma}\left(k_{0}, m_{0}, v_{0}\right)$ recursively as follows. At date $t=0$, for each $x_{0} \in[0,1]$, define

$$
\left(\tau_{k, 0}^{*}, \tau_{\ell, 0}^{*}\right)\left(x_{0}\right):=\left(A_{1}, A_{2}\right)\left(k_{0}, m_{0}, v_{0}\right)\left(x_{0}\right)
$$

and let

$$
\begin{aligned}
\hat{\sigma}_{G}\left(k_{0}, m_{0}, v_{0}\right)(0)\left(x_{0}\right) & =\left(\tau_{k, 0}^{*}, \tau_{\ell, 0}^{*}\right)\left(x_{0}\right) \text { and } \\
\hat{\sigma}_{C}\left(k_{0}, m_{0}, v_{0}\right)(0)\left(x_{0}, \tau_{k, 0}^{*}\left(x_{0}\right), \tau_{\ell, 0}^{*}\left(x_{0}\right)\right) & :=\left(A_{3}, A_{4}, A_{5}\right)\left(k_{0}, m_{0}, v_{0}\right)\left(x_{0}\right) .
\end{aligned}
$$

For $\left(\tau_{k, 0}, \tau_{\ell, 0}\right) \neq\left(\tau_{k, 0}^{*}, \tau_{\ell, 0}^{*}\right)\left(x_{0}\right)$, let

$$
\hat{\sigma}_{C}\left(k_{0}, m_{0}, v_{0}\right)(0)\left(x_{0}, \tau_{k, 0}, \tau_{\ell, 0}\right):=W E^{*}\left(k_{0}, \tau_{k, 0}, \tau_{\ell, 0}\right),
$$

where $W E^{*}$ was introduced in the definition of admissibility. This specifies the period $t=0$ strategies. For $t=1$ and arbitrary history $h^{0}$, let $k_{1}\left(h^{0}\right):=\kappa\left(k_{0}, h^{0}\right)$, and define $\left(m_{1}, v_{1}\right)\left(h^{0}\right)$

\footnotetext{
${ }^{11}$ By Caratheodory's theorem, the functions can in fact be assumed to be piecewise constant, taking on only three values. Since $(m, v) \in \operatorname{co}\left(B^{R}(W)\right)$, there exist three pairs $\left(m_{j}, v_{j}\right) \in B^{R}(W)$ and corresponding weights $\alpha_{j} \in[0,1], j=1,2,3$, such that $(m, v)=\sum \alpha_{i}\left(m_{i}, v_{i}\right)$ and $\alpha_{1}+\alpha_{2}+\alpha_{3}=1$. Select three admissible tuples $\xi^{j} \in \mathbf{R}^{7}$ such that $\Psi\left(\xi^{j}\right)=\left(m_{j}, v_{j}\right), j=1,2,3$, and let $I_{1}:=\left[0, \alpha_{1}\right], I_{2}:=\left(\alpha_{1}, \alpha_{1}+\alpha_{2}\right), I_{3}:=\left[\alpha_{1}+\alpha_{2}, 1\right]$. Then we can make $\left(\tau_{k}(x), \tau_{\ell}(x), \ell(x), c(x), k_{+}(x), m_{+}(x), v_{+}(x)\right)=\xi^{j}$ for each $x \in I_{j}$.
} 
as follows. If neither households nor the government has observably deviated: $\left(\tau_{k, 0}, \tau_{\ell, 0}\right)=$ $\left(\tau_{k, 0}^{*}, \tau_{\ell, 0}^{*}\right)\left(x_{0}\right)$ and $\left(\ell_{0}, c_{0}, k_{1}\right)=\left(A_{3}, A_{4}, A_{5}\right)\left(k_{0}, m_{0}, v_{0}\right)\left(x_{0}\right)$, let

$$
\left(m_{1}, v_{1}\right)\left(h^{0}\right):=\left(A_{6}, A_{7}\right)\left(k_{0}, m_{0}, v_{0}\right)\left(x_{0}\right) .
$$

If $\left(\tau_{k, 0}, \tau_{\ell, 0}\right) \neq\left(\tau_{k, 0}^{*}, \tau_{\ell, 0}^{*}\right)\left(x_{0}\right)$ but $\left(\ell_{0}, c_{0}, k_{1}\right)=\left(A_{3}, A_{4}, A_{5}\right)\left(k_{0}, m_{0}, v_{0}\right)\left(x_{0}\right)$ (the government deviated but the households followed the equilibrium response to the deviation), let

$$
\left(m_{1}, v_{1}\right)\left(h^{0}\right)=\left(M^{*}\left(k_{0}, \tau_{k, 0}, \tau_{\ell, 0}\right), \underline{W}\left(k_{1}, M^{*}\left(k_{0}, \tau_{k, 0}, \tau_{\ell, 0}\right)\right)\right),
$$

where, again, $M^{*}$ is introduced in the definition of admissibility. Otherwise, let $\left(m_{1}, v_{1}\right)\left(h^{0}\right)$ be an arbitrary element of $W\left(k_{1}\right)$. (It doesn't matter which element is chosen since this case requires the simultaneous deviation of a positive fraction of agents, and as we discussed earlier, such a case is irrelevant for checking households' incentives.) Note that since $A\left(k_{0}, m_{0}, v_{0}\right)\left(x_{0}\right)$ is an admissible tuple w.r.t. $W$ by construction and $W \subset B(W)$ by assumption, we have that $\left(k_{1}, m_{1}, v_{1}\right)\left(h^{0}\right) \in \operatorname{graph}(B(W))$, and thus $A\left(\left(k_{1}, m_{1}, v_{1}\right)\left(h^{0}\right)\right)$ is also well defined. Hence, we can define the actions for the government and the households at $t=1$ for each $\left(k_{1}, m_{1}, v_{1}\right)\left(h^{0}\right)$, $h_{0} \in H\left(k_{0}, 0\right)$ in a similar fashion. Continuing this way, we can roll out the entire strategy $\hat{\sigma}\left(k_{0}, m_{0}, v_{0}\right)$.

This recursive procedure effectively defines a family of symmetric strategy profiles, one for each $\left(k_{0}, m_{0}, v_{0}\right) \in \operatorname{graph}(B(W))$. We first show that each $\hat{\sigma}\left(k_{0}, m_{0}, v_{0}\right)$ is an SSE. Note that for any $\left(k_{0}, m_{0}, v_{0}\right) \in \operatorname{graph}(B(W))$, period $t$, and history $h^{t-1}$, there exists $\left(k^{\prime}, m^{\prime}, v^{\prime}\right) \in$ $\operatorname{graph}(B(W))$ such that the continuation strategy induced by $\hat{\sigma}\left(k_{0}, m_{0}, v_{0}\right)$ after $h^{t-1}$ is $\hat{\sigma}\left(k^{\prime}, m^{\prime}, v^{\prime}\right)$. Therefore, to check that the households' incentive constraints (condition (2) of an SSE) are satisfied, it is enough to check that for each $\left(k_{0}, m_{0}, v_{0}\right) \in \operatorname{graph}(B(W))$, if $\left\{\left(\tau_{k, t}, \tau_{\ell, t}, \ell_{t}, c_{t}, k_{t+1}\right)\right\}_{t \geq 0}$ is the outcome path of $\hat{\sigma}\left(k_{0}, m_{0}, v_{0}\right)$, then $\left\{\left(\ell_{t}, c_{t}, k_{t+1}\right)\right\}_{t \geq 0}$ is a 
Walrasian equilibrium of $\Gamma_{W}^{R}(k, \tau)$, where $\tau=\left\{\left(\tau_{k, t}, \tau_{\ell, t}\right)\right\}_{t \geq 0}$. But, by condition (1) of admissibility, the consumption path satisfies the Euler conditions for problem $\left(P^{R}\right)$, and therefore, by Lemma $2^{\prime}$, it is indeed a Walrasian equilibrium for $\Gamma_{W}^{R}(k, \tau)$.

We next show that $\left(m_{0}, v_{0}\right)=\Phi\left(k_{0}, \hat{\sigma}\left(k_{0}, m_{0}, v_{0}\right)\right)$ for all $\left(k_{0}, m_{0}, v_{0}\right) \in \operatorname{graph}(B(W))$. Fix $\left(k_{0}, m_{0}, v_{0}\right) \in \operatorname{graph}(B(W))$, and for each $x_{0} \in[0,1]$, let

$$
\begin{aligned}
& \left(\tau_{k, 0}, \tau_{\ell, 0}, \ell_{0}, c_{0}, k_{1}, m_{1}, v_{1}\right)\left(x_{0}\right)=A\left(k_{0}, m_{0}, v_{0}\right)\left(x_{0}\right), \text { and } \\
& G\left(x_{0}\right)=\tau_{k, 0}\left(x_{0}\right) f_{k}\left(k_{0}, \ell_{0}\left(x_{0}\right)\right) k_{0}+\tau_{\ell, 0}\left(x_{0}\right) f_{\ell}\left(k_{0}, \ell_{0}\left(x_{0}\right)\right) \ell_{0}\left(x_{0}\right) .
\end{aligned}
$$

By construction

$$
\begin{aligned}
v_{0} & =\int_{0}^{1}\left\{(1-\beta)\left[u\left(\ell_{0}\left(x_{0}\right), c_{0}\left(x_{0}\right)\right)+g\left(G\left(x_{0}\right)\right)\right]+\beta v_{1}\left(x_{0}\right)\right\} d x_{0}, \text { and } \\
m_{0} & =\int_{0}^{1}\left(1-\tau_{k, 0}^{*}\left(x_{0}\right)\right) f_{k}\left(k_{0}, \ell_{0}\left(x_{0}\right)\right) u_{c}\left(\ell_{0}\left(x_{0}\right), c_{0}\left(x_{0}\right)\right) d x_{0}=\Phi_{C}\left(k_{0}, \hat{\sigma}\left(k_{0}, m_{0}, v_{0}\right)\right) .
\end{aligned}
$$

Also,

$$
\begin{aligned}
\Phi_{G}\left(k_{0}, \hat{\sigma}\left(k_{0}, m_{0}, v_{0}\right)\right)= & \int_{0}^{1}\left\{(1-\beta)\left[u\left(\ell_{0}\left(x_{0}\right), c_{0}\left(x_{0}\right)\right)+g\left(G\left(x_{0}\right)\right)\right]\right. \\
& \left.+\beta \Phi_{G}\left(k_{1}\left(x_{0}\right), \hat{\sigma}\left(k_{1}\left(x_{0}\right), m_{1}\left(x_{0}\right), v_{1}\left(x_{0}\right)\right)\right)\right\} d x_{0} .
\end{aligned}
$$

Hence,

$$
\left|v_{0}-\Phi_{G}\left(k_{0}, \hat{\sigma}\left(k_{0}, m_{0}, v_{0}\right)\right)\right| \leq \max _{\left(k_{+}, m_{+}, v_{+}\right) \in \operatorname{graph}(W)} \beta\left|v_{+}-\Phi_{G}\left(k_{+}, \hat{\sigma}\left(k_{+}, m_{+}, v_{+}\right)\right)\right| .
$$

Since $W \subset B(W)$ and this inequality holds for all $\left(k_{0}, m_{0}, v_{0}\right) \in B(W)$, 


$$
\begin{aligned}
& \max _{\left(k_{0}, m_{0}, v_{0}\right) \in \operatorname{graph}(B(W))}\left|v_{0}-\Phi_{G}\left(k_{0}, \hat{\sigma}\left(k_{0}, m_{0}, v_{0}\right)\right)\right| \\
\leq & \beta\left[\max _{\left(k_{+}, m_{+}, v_{+}\right) \in \operatorname{graph}(B(W))}\left|v_{+}-\Phi_{G}\left(k_{+}, \hat{\sigma}\left(k_{+}, m_{+}, v_{+}\right)\right)\right|\right] .
\end{aligned}
$$

Therefore, $v_{0}=\Phi_{G}\left(k_{0}, \hat{\sigma}\left(k_{0}, m_{0}, v_{0}\right)\right)$ for all $\left(k_{0}, m_{0}, v_{0}\right) \in \operatorname{graph}(B(W))$.

Finally, condition (3) of admissibility implies that the government constraints (condition (1) of an SSE) are satisfied.

We next prove two lemmas, which combined establish Theorem 2. Fix once and for all an arbitrary usc value correspondence $W$. Since $\operatorname{graph}(W)$ is compact, there exist $\underline{\underline{v}}, \overline{\bar{v}} \in \mathbf{R}$, such that

$$
W(k) \in[0, \overline{\bar{m}}] \times[\underline{\underline{v}}, \overline{\bar{v}}] \quad \text { for all } k \in[\underline{k}, \bar{k}] .
$$

Lemma 7. $\operatorname{graph}\left(B^{R}(W)\right)$ is bounded.

Proof. Pick any $k \in[\underline{k}, \bar{k}]$ and any tuple $\xi=\left(\tau_{k}, \tau_{\ell}, \ell, c, k_{+}, m_{+}, v_{+}\right)$admissible w.r.t. $W$ at $k$. From Lemma 3 (Lemma $\left.3^{\prime}\right)$, we have that $\Psi_{C}(k, \xi) \in[0, \overline{\bar{m}}]$.

Since $\xi$ is an admissible tuple w.r.t. $W,\left(\ell, c, k_{+}\right)$must solve $\left(P_{t}\right)$ with parameters $\left(k, \tau_{k}, \tau_{\ell}, m_{+}\right)$(and $p_{k}=f_{k}(k, \ell)$ and $\left.p_{\ell}=f_{\ell}(k, \ell)\right)$. Since $m_{+} \geq 0$, and since not working, not consuming, and not saving is feasible, the objective function of $\left(P_{t}\right)$ can be no lower than $u(0,0)$ (which is finite by assumption). This implies that

$$
u(\ell, c) \geq u(0,0)-\beta \overline{\bar{m}} \bar{k} .
$$

Hence $\Psi_{G}(k, \xi) \in\left[\underline{\underline{v}}_{+}, \overline{\bar{v}}_{+}\right]$, where

$$
\begin{aligned}
& \underline{\underline{v}}_{+}:=\quad(1-\beta)[u(0,0)-\beta \overline{\bar{m}} \bar{k}+g(0)]+\beta \underline{\underline{v}} \\
& \overline{\bar{v}}_{+}:=\quad(1-\beta)[u(0, \bar{k})+g(\bar{\tau} \bar{k})]+\beta \overline{\bar{v}} .
\end{aligned}
$$


Therefore, $\operatorname{graph}\left(B^{R}(W)\right) \subset[\underline{k}, \bar{k}] \times[0, \overline{\bar{m}}] \times\left[\underline{\underline{v}}_{+}, \overline{\bar{v}}_{+}\right]$.

Lemma 8. $\operatorname{graph}\left(B^{R}(W)\right)$ is closed.

Proof. Pick any sequence $\left\{\left(k^{\alpha}, w^{\alpha}\right)\right\}_{\alpha}$ such that $w^{\alpha} \in B^{R}(W)\left(k^{\alpha}\right)$ for each $\alpha$ and $\left(k^{\alpha}, w^{\alpha}\right) \rightarrow$ $\left(k^{*}, w^{*}\right)$. By definition, for each $\alpha$ there exists a tuple $\xi^{\alpha}=\left(\tau_{k}^{\alpha}, \tau_{\ell}^{\alpha}, \ell^{\alpha}, c^{\alpha}, k_{+}^{\alpha}, m_{+}^{\alpha}, v_{+}^{\alpha}\right)$ admissible w.r.t. $W$ at $k^{\alpha}$, such that

$$
w^{\alpha}=\left(w_{C}^{\alpha}, w_{G}^{\alpha}\right)=\Psi\left(k^{\alpha}, \xi^{\alpha}\right) .
$$

Since $\left\{\xi^{\alpha}\right\}_{\alpha} \subset[\underline{\tau}, \bar{\tau}]^{2} \times[0,1] \times[\underline{k}, \bar{k}]^{2} \times[0, \overline{\bar{m}}] \times[\underline{\underline{v}}, \overline{\bar{v}}]$, we can assume without loss of generality that this sequence converges to a tuple $\xi^{*}=\left(\tau_{k}^{*}, \tau_{\ell}^{*}, \ell^{*}, c^{*}, k_{+}^{*}, m_{+}^{*}, v_{+}^{*}\right)$. By continuity, it is easy to verify that $\xi^{*}$ is admissible w.r.t. $W$ at $k^{*}$ and

$$
w^{*}=\Psi\left(k, \xi^{*}\right) .
$$

Hence, $\left(k^{*}, w^{*}\right) \in \operatorname{graph}\left(B^{R}(W)\right)$, and $\operatorname{graph}\left(B^{R}(W)\right)$ is closed.

The previous two lemmas establish that $\operatorname{graph}\left(B^{R}(W)\right)$ is compact. Hence, $B^{R}(W)$ is usc. Since $B(W)(k)=\operatorname{co}\left(B^{R}(W)(k)\right)$, we also have that $B(W)(k)$ is compact for all $k \in[\underline{k}, \bar{k}]$, and that graph $(B(W))$ is compact. Therefore, $B(W)$ is usc, which establishes Theorem 2.

Algorithm: We now discuss practical aspects of the algorithm's implementation. The first issue we need to confront is the representation of the graph of a value correspondence $W$. In general a precise description of a set is not (numerically) possible. We use the fact that each $W(k), k \in[\underline{k}, \bar{k}]$, is a closed convex set, and thus $W(k)$ is equal to the intersection of 
all closed half-spaces that contain it. Hence, there exists a function $a:[\underline{k}, \bar{k}] \times[-\pi, \pi) \rightarrow \mathbf{R}$ such that

$$
W(k)=\{(m, v) \mid \cos (\theta) m+\sin (\theta) v \leq a(k, \theta) \text { for all } \theta \in[-\pi, \pi)\} .
$$

The value $a(k, \theta)$ is the distance from the origin of the supporting hyperplane whose normal is the unitary vector $(\cos (\theta), \sin (\theta))$.

In the numerical algorithm, we define a grid $\left\{k_{1}, \ldots, k_{I}\right\} \times\left\{\theta_{1}, \ldots, \theta_{J}\right\}$ on $[\underline{k}, \bar{k}] \times$ $[-\pi, \pi)$ and construct the matrix $A=\left[a_{i j}\right]$, where $a_{i j}=a\left(k_{i}, \theta_{j}\right)$. Since the grid is finite, we only get an "outer" approximation for each set $W\left(k_{i}\right)$ :

$$
W\left(k_{i}\right) \subset\left\{(m, v) \mid \cos \left(\theta_{j}\right) m+\sin \left(\theta_{j}\right) v \leq a_{i j} \text { for all } j=1, \ldots, J\right\} .
$$

For intermediate values of $k$ we use (linear) interpolation to obtain an approximation of $W(k)$.

The numerical implementation of the map $B$ then takes an $I \times J$ matrix $A$, representing a correspondence $W$, into another matrix representing $B(W)$. To simplify the algorithm, we only consider values of $\left(\tau_{k}, \tau_{\ell}\right)$ on a given finite grid (this can be viewed as a genuine policy restriction), and values of $m_{+}$in another finite grid.

\section{References}

[1] Abreu, D. 1986. "Extremal Equilibria of Oligopolistic Supergames," Journal of Economic Theory 39: 191-228.

[2] Abreu, D. 1988. "Towards a Theory of Discounted Repeated Games," Econometrica 56: 383-96.

[3] Abreu, D., D. Pearce, and E. Stacchetti. 1986. "Optimal Cartel Equilibrium with Imperfect Monitoring," Journal of Economic Theory 39: 251-69. 
[4] Abreu, D., D. Pearce, and E. Stacchetti. 1990. "Towards a Theory of Discounted Repeated Games with Imperfect Monitoring," Econometrica 58: 1041-64.

[5] Atkeson, A. 1991. "International Lending with Moral Hazard and Risk of Repudiation," Econometrica 59: 1069-90.

[6] Benhabib, J. and A. Rustichini. 1997. "Optimal Taxes Without Commitment," Journal of Economic Theory 77(2): 231-59.

[7] Berge, C. 1963. Topological Spaces. New York: Macmillan.

[8] Chamley, C. 1986. "Optimal Taxation of Capital Income in General Equilibrium with Infinite Lives," Econometrica 54: 607-22.

[9] Chang, R. 1998. "Credible Monetary Policy with Long Lived Agents: Recursive Approaches," Journal of Economic Theory 81(2): 431-67.

[10] Chari, V. and P. Kehoe. 1990. "Sustainable Plans," Journal of Political Economy 98: 783-802.

[11] Chari, V. and P. Kehoe. 1993a. "Sustainable Plans and Debt," Journal of Economic Theory 61: 230-61.

[12] Chari, V. and P. Kehoe. 1993b. "Sustainable Plans and Mutual Default," Review of Economic Studies 60: 175-95.

[13] Conklin, J. and K. Judd. 1996. "Computing Value Correspondences for Two-Player Games with State Variables," mimeo, Hoover Institution. 
[14] Judd, K. 1985. "Redistributive Taxation in a Simple Perfect Foresight Model," Journal of Public Economics 28: 59-83.

[15] Kydland, F. and E. Prescott. 1977. "Rules Rather than Discretion: The Inconsistency of Optimal Plans," Journal of Political Economy 87: 473-92.

[16] Pearce, D. 1987. "Renegotiation-Proof Equilibria: Collective Rationality and Intertemporal Cooperation," Cowles Foundation Discussion Paper No. 855.

[17] Persson, M., T. Persson, and L.E.O. Svensson. 1987. "Time Consistency of Fiscal and Monetary Policy," Econometrica 5: 1419-31.

[18] Rogoff, K. 1989. "Reputation, Coordination, and Monetary Policy," in Modern Business Cycle Theory, ed. R. Barro. Cambridge: Harvard University Press.

[19] Stokey, N. 1989. "Reputation and Time Consistency," American Economic Review 79: $134-39$.

[20] Stokey, N. 1991. "Credible Public Policy," Journal of Economic Dynamics and Control 15: $627-57$. 